\title{
BERNARD LASCAR
}

\section{RICHARD LASCAR}

\section{NICOLAS LERNER}

\section{Propagation of Singularities for Non Real Pseudo-Differential Operators}

Publications de l'Institut de recherche mathématiques de Rennes, 1992-1993, fascicule 1 «Fascicule d'équations aux dérivées partielles », , exp. n 5, p. 1-25

(C) Département de mathématiques et informatique, université de Rennes, 1992-1993, tous droits réservés.

L'accès aux archives de la série « Publications mathématiques et informatiques de Rennes » implique l'accord avec les conditions générales d'utilisation (http://www.numdam.org/conditions). Toute utilisation commerciale ou impression systématique est constitutive d'une infraction pénale. Toute copie ou impression de ce fichier doit contenir la présente mention de copyright.

\section{Numdam}




\title{
Propagation of singularities for non real pseudo-differential operators
}

\author{
Bernard Lascar \\ Mathématiques. URA 213, 4 Place Jussieu 75252 Paris Cedex 05. \\ Richard Lascar \\ Mathématiques. URA 213, 4 Place Jussieu 75252 Paris Cedex 05. \\ Nicolas Lerner \\ Mathématiques. URA 305.Université de Rennes 1. \\ Campus de Beaulieu. 35042 Rennes Cedex.
}

January 1993 


\begin{abstract}
The purpose of this work is to prove a theorem of propagation of singularities for a class of non real pseudo-differential operator with multiple characteristics. The main tools are $L^{2}$ estimates on the time dependent Schrödinger equation related to $P$. We extend here the results of $[6]$; we improve the results announced by the second author in [7].

The second part of this work consists in an extension of the result of [5] to complex valued symbols.
\end{abstract}




\section{Contents}

I The General result. 1

1 Introduction and main statement 1

2 Estimates on solutions of the Schrödinger equation 4

3 Semi global $L^{2}$ estimates. 11

II A more precise result in a particular case. 13

4 Construction of the stable manifolds. 13

5 The energy estimate. 19

5.1 The basic $L^{2}$ inequality. . . . . . . . . . . 19

5.2 Concatenations. . . . . . . . . . . . . 21

\section{Part I}

\section{The General result.}

We start by a general result which could not be optimal in all the cases scanned here. The approach is similar to [6] but we use also time dependent $L^{2}$ estimate and some informations on the parametrix constructed in [6]. The main difference with the proof in [7] is that we need to have an analysis of the microlocal structure of the parametrix of the time dependent Schrödinger equation associated with the self-adjoint part of our operator.

\section{Introduction and main statement}

Let $P\left(x, \lambda^{-1} D_{x}, \lambda\right)$ be a pseudo-differential operator depending on a large parameter $\lambda$, defined by the Weyl formula : 


$$
\begin{aligned}
P\left(x, \lambda^{-1} D_{x}, \lambda\right) u(x, \lambda) & =o p_{1 / 2}((p(x, \xi / \lambda, \lambda))(u)(x) \\
& =\left(\frac{\lambda}{2 \pi}\right)^{n} \int p\left(\frac{x+y}{2}, \xi, \lambda\right) e^{i \lambda(x-y) \xi_{u}(y, \lambda) d y d \xi}
\end{aligned}
$$

We shall write the operator given by formula $I(p(x, \xi, \lambda))^{w_{\lambda}}$.

The full symbol $p(x, \xi, \lambda)$ has an expansion as

$$
p(x, \xi, \lambda)=p_{1}(x, \xi)+i p_{2}(x, \xi)+\lambda^{-1} p_{0}(x, \xi, \lambda)
$$

where $p_{1}$ and $p_{2}$ are real and $p_{2} \geq 0 . p_{0}(x, \xi, \lambda)$ is a zero order symbol i.e. satisfies estimates :

$$
\text { For all multi-indices } \alpha \text { and } \beta\left|D_{x}^{\alpha} D_{\xi}^{\beta} p_{0}(x, \xi, \lambda)\right| \leq C_{\alpha, \beta} \text {. }
$$

It is a consequence of formulas 1 and 2 that

$$
P\left(x, \lambda^{-1} D_{x}, \lambda\right)=P_{1}\left(x, \lambda^{-1} D_{x}, \lambda\right)+i P_{2}\left(x, \lambda^{-1} D_{x}, \lambda\right)
$$

where $P_{1}$ and $P_{2}$ are self-adjoint pseudo-differential operators with symbols respectively

$$
\begin{aligned}
& P_{1}=\left(p_{1}(x, \xi)+\lambda^{-1} \operatorname{Re}\left(p_{0}(x, \xi, \lambda)\right)^{w_{\lambda}}\right. \\
& P_{2}=\left(p_{2}(x, \xi)+\lambda^{-1} \operatorname{Im}\left(p_{0}(x, \xi, \lambda)\right)^{w_{\lambda}}\right.
\end{aligned}
$$

We shall make $L^{2}$ estimates on the solutions $u(t)$ of the Schrödinger equation $\left(D_{t}+P\left(x, \lambda^{-1} D_{x}, \lambda\right)\right) u(t)=0$. We shall therefore use some constructions made in [6]. We need to recall the hypotheses of this work.

- $(H)_{1}$ : Let $\Phi_{t}$ be the bicharacteristic flow of $p_{1}$ at the time $t$. Let $\rho_{0}$ in $T^{*} \mathbf{R}^{n}$ be a point near which we shall work. Let $h(t) \in o(t)$ when $t \rightarrow \infty, h \geq 0$ a function, and $W$ a neighborhood of $\rho_{0}$ such that any bicharacteristic curve of $p_{1}$ with end points lying in

$$
\Lambda(W, h)=\left\{\begin{array}{c}
\left(\rho_{1}, \rho_{2}\right) \in W^{2} ; \exists t \geq 0 \text { such that } \rho_{1}=\Phi_{t}\left(\rho_{2}\right), \\
\text { and if } 0 \leq s \leq t \Phi_{s}\left(\rho_{2}\right) \in W \text { and }\left|p_{1}\left(\rho_{1}\right)\right| \leq \exp (-h(t))
\end{array}\right\}
$$

is $N_{0}$ admissible ${ }^{1}$ for a function $\varepsilon \in[0,1] \rightarrow N_{0}(\varepsilon) \in \mathbf{R}^{+}$. We refer to [6] pp 468-469 for a definition and for sufficient conditions which

\footnotetext{
${ }^{\mathrm{a}}$ A curve $t \in[0, T] \rightarrow \gamma(t) \in W$ is $N_{0}$ admissible if for any $\varepsilon>0$, there exists a partition of $[0, T]$ in intervals of type $I$ and $\mathcal{J}$, the number of these intervalls is less than $N_{0}(\epsilon)$. An interval of type $I$ remains at a distance less than $\epsilon$ from a point in the double characteristic set of $P_{1}$, an interval of type $\mathcal{J}$ has a lenght less than $N_{0}(\varepsilon)$.
} 
imply this property. We shall not recall here the details, but we just mention that it is satisfied if the bicharacteristics of $p_{1}$ whose lenght is large enough leave a neighborhood of $\left(\rho_{0}, \rho_{0}\right)$.

Let $N=\left\{\rho \in \mathrm{T}^{*}\left(\mathrm{R}^{n}\right) ; p_{1}(\rho)=d p_{1}(\rho)=0\right\}$ be the set of double characteristics of $P_{1}$.

- $(\mathrm{H})_{2}$ : The main assumption is that on $N$, the dimension of the space spanned by the generalized eigenvectors associated with ejgenvalues of positive imaginary part is constant.

- $(\mathrm{H})_{3}$ : On $N, I m p_{0}(\rho)>0$. This inequality means that $I m p_{0}$ has a positive lower bound with respect to $\rho$ and $\lambda$.

Let us define

$$
C(\bar{W})=\bigcap_{h \in \omega} \overline{\Lambda(W, h)}
$$

where $\omega$ is the set of all non negative increasing functions $h(t) \in o(t)$ when $t \rightarrow \infty$.

We consider $C(\bar{W})$ as a relation in $T^{*}\left(\mathbf{R}^{n}\right)$.

We note by $O F(u)$ the oscillatory front set of a bounded family of tempered distributions $u(x, \lambda)$.

Let us recall that we say that $\left(x_{0}, \xi_{0}\right) \in(O F(u))^{c}$ if there are neighborhoods $V$ of $x_{0}$ and $L$ of $\xi_{0}$ such that for any $\varphi \in \mathrm{C}_{0}^{\infty}(V)$

$$
\text { For all } N \in \mathrm{N} \text {, for } \lambda \geq 1 \sup _{\xi \in L}|\widehat{\varphi v}|(\lambda \xi, \lambda) \leq C_{N} \lambda^{-N} \text {. }
$$

The main result can now be stated.

Theorem 1 Assume that the assumptions $(\mathrm{H})_{1}(\mathrm{H})_{2},(\mathrm{H})_{3}$ are satisfied for a suitable set $W$ and for some function $h_{0} \in \omega$. Let $u(x, \lambda)$ be a bounded family of tempered distributions. If $O F(P u) \cap \bar{W}=0$ and $C(\bar{W})(\rho) \cap \partial W \cap$ $O F(u)=\emptyset$, then $\rho \notin O F(u)$.

We easily deduce :

Corollary 1 Let $\rho_{0} \in \mathrm{T}^{*}\left(\mathbf{R}^{n}\right) \backslash 0, P\left(x, D_{x}\right)$ a pseudo-differential operator in the usual sense. Assume that $(\mathrm{H})_{1}(\mathrm{H})_{2},(\mathrm{H})_{3}$ are satisfied for a neighborhod $W$ of $\rho_{0}$ and $a$ function $h_{0} \in \omega$. Let $u$ be a distribution such that $W F(P u) \cap$ $\bar{W}=\emptyset$ and $C(\bar{W})(\rho) \cap \partial W \cap W F(u)=\emptyset$, then $\rho \notin W F(u)$. 
The main difference between the proof of this theorem and the corresponding result in [7] is the presence in the bicharacteristic flow of $p_{1}$ of expansive diractions. This will make us to use fully the construction of the parametrix of [6] instead of using only microlocalisations in a semi-global $L^{2}$ inequality for the solutions of the time dependent Schrödinger equation associated with $P_{1}$.

\section{Estimates on solutions of the Schrödinger equa- tion}

We shall work with a family of solutions of the Schrödinger equation $\left(D_{t}+\right.$ $\left.P\left(x, \lambda^{-1} D_{x}\right)\right) u(t)=0$, where $D_{t}=(1 / i \lambda) \partial / \partial t$.

We need to make a Fourier-Bros-Iagolnitzer transformation (see [9] and [6]). Let

$$
T u(x, \lambda)=\left(\frac{\lambda}{2 i \pi}\right)^{n / 2} \int e^{-\lambda / 2\left((x-y)^{2}-x^{2} / 2\right)} u(y, \lambda) d y
$$

this is a unitary transformation from $L^{2}\left(\mathbf{R}^{n}\right)$ to the space $H_{\varphi_{0}}\left(\mathbf{C}^{n}\right)$ of entire functions in $L^{2}\left(C^{n}, e^{-2 \lambda \varphi_{0}} \mathrm{~L}(d x)\right)$, where $L(d x)$ is the Lebesgue measure in $\mathrm{C}^{n}$, and $\varphi_{0}(x)=\frac{1}{4}|x|^{2}$.

We note by the same letter an operator and its conjugate by $T$.

We have a Bergman projector from $L^{2}\left(C^{n}, e^{-2 \lambda \varphi_{0}} \mathrm{~L}(d x)\right)$ to $H_{\varphi_{0}}\left(\mathbf{C}^{n}\right)$ given by the formula

$$
S u(x, \lambda)=\left(\frac{\lambda}{2 i \pi}\right)^{n} \int e^{\lambda x \tilde{y} / 2} u(y, \lambda) e^{-2 \lambda \varphi_{0}(y)} \mathrm{L}(d y)
$$

see [9] for these formulas. Let us say that the formula 9 is obtained by integrating the formal integral in $T T^{-1}$ along a suitable contour.

In [6] we can find some constructions for an approximate solution $E_{t} u(x, \lambda)$ of the equation $\left(D_{t}+P_{1}\left(x, \lambda^{-1} D_{x}\right)\right)\left(E_{t} u\right) \equiv 0 ;\left.E_{t} u\right|_{t=0} \equiv u$, we shall make this more precise later.

$$
E_{t} u(x, \lambda)=\left(\frac{\lambda}{2 i \pi}\right)^{n} \int e^{i \lambda \psi(t, x, \bar{y})} e(t, x, \bar{y}, \lambda) \chi(t, x, \bar{y}) u(y, \lambda) e^{-2 \lambda \varphi_{0}(y)} L(d y)
$$

where $\varphi(t, x, y)$ is a solution of the phase equation with value $\varphi(0, x, \bar{y})=$ $-i x \bar{y} / 2 ; e(t, x, y, \lambda)$ is a solution of transport equations, $\chi(t, x, y)$ is a cut-off function. 
Let

$$
\Gamma_{t}(W, h)=\left\{(x, y) ;\left(x, \frac{2}{i} \frac{\partial \varphi_{0}}{\partial x}, y, \frac{2}{i} \frac{\partial \varphi_{0}}{\partial y}\right) \in \Lambda_{t}^{\prime}(W, h)\right\}
$$

where $\Lambda_{t}^{\prime}(W, h)$ is the image by the complex canonical transformation generated by $\varphi_{0}(x)$ of the Lagrangean sub manifold

$$
\Lambda_{t}(W, h)=\left\{\begin{array}{c}
\left(\rho_{1}, \rho_{2}^{\prime}\right) \in W^{2} ; \text { such that } \rho_{1}=\exp \left(t H_{p_{1}}\right)\left(\rho_{2}\right) \text { if } 0 \leq s \leq t \\
\exp \left(s H_{p_{1}}\right)\left(\rho_{2}\right) \in W \text { and }\left|p_{1}\left(\rho_{1}\right)\right| \leq \exp (-h(t))
\end{array}\right\}
$$

where $\rho_{2}^{\prime}$ is the antipodal point of $\rho_{2}$.

$\Gamma_{t}(W, h)$ is totally real in $\mathbf{C}^{n} \times \mathbf{C}^{n}$. We again refer to [6] for the construction of a convenient projection $\mathbf{C}^{n} \times \mathbf{C}^{n} \rightarrow \Gamma_{t}(W, h) ; z \rightarrow m(t, z) \in$ $\Gamma_{t}(W, h) ; m(t, z)$ will be defined uniquely by the additional property that $z-m(t, z) \in i \Gamma_{m(t, z)} \Gamma_{t}(W, h)$.

Therefore functions on $\Gamma_{t}(W, h)$ give rise to almost analytic extension in $\mathbf{C}^{n} \times \mathbf{C}^{n}$. Appropriate controls with respect to $t$ are obtained in [6]. From these controls it follows that all derivatives with respect to $(t, x)$ of these maps are bounded by some $\exp (h(t))$ with a function $h(t) \in o(t)$ when $t \rightarrow \infty$. So $\varphi(t, x, y), \ldots, \chi(t, x, y)$ will be almost analytic on $\Gamma_{t}$ and there derivatives are bounded by some $\exp (h(t))$. A more precise decay in time for the amplitude $e(t, x, y)$ is obtained and this will be discussed later since this point is essential in our discussion.

Proposition 1 There are two constants $C>0, \gamma>0$ such that

$$
\left\|E_{t}\right\| \leq C \lambda^{n} \exp (-1 / 2 \gamma t)
$$

$\gamma$ being as close as we wish of the lower bound of

$$
T^{+}(\rho)=\sum_{z_{j} \in \operatorname{Spec}\left(F_{p_{1}}\right), \operatorname{Rez} j>0} \operatorname{Re} z_{j} .
$$

We shall not use this result here, but it is woth mentionning since it is the key point of the proof in [6]. The proof will be derived from elements of the proof of the following Proposition.

Proposition 2 There are constants $M>0, C>0$ such that

$$
\left\|E_{t}\right\| \leq C
$$

if $\exp (M t) \leq \lambda$, the norms are taken in $H_{\varphi_{0}}\left(\mathrm{C}^{n}\right)$. 
Proof. Let us prove Proposition 2. Let $E_{t}^{*}$ be the adjoint of $E_{t}$ in $H_{\varphi_{0}}\left(\mathrm{C}^{n_{0}}\right)$. If we write $d \mu(x)=e^{-2 \lambda \varphi_{0}(x)} \mathrm{L}(d x)$ the kemel (with respect to u) of $E_{t}^{*}$ is given by $E_{t}^{*}(x, y)=\int S(x, z) \overline{E_{t}(y, z)} d \mu(z)$, the kernel of $E_{t}^{*} E_{t}$ is therefore $E_{t}^{*} E_{t}(x, y)=\int S\left(x, x_{1}\right) E_{t}\left(x_{2}, x_{1}\right) E_{t}\left(x_{2}, y\right) d \mu\left(x_{1}\right) d \mu\left(x_{2}\right)$. We write this integral

$$
E_{t}^{*} E_{t}(x, y)=\int e^{i \lambda H\left(t, x, y ; x_{1}, x_{2}\right)} f\left(t, x, y ; x_{1} x_{2}, \lambda\right) d \mu\left(x_{1}\right) d \mu\left(x_{2}\right)
$$

In 15 we have

$$
H\left(t, x, y ; x_{1}, x_{2}\right)=\psi\left(x, x_{1}\right)-\overline{\varphi\left(t, x_{2}, \bar{x}_{1}\right)}+\varphi\left(t, x_{2}, \bar{y}\right)+2 i\left(\varphi_{0}\left(x_{1}\right)+\varphi_{0}\left(x_{2}\right)\right)
$$

and

$$
f\left(t, x, y ; x_{1}, x_{2}, \lambda\right)=c \lambda^{3 n} \overline{e\left(t, x_{2}, \bar{x}_{1}\right)} e\left(t, x_{2}, \bar{y}\right) \overline{\chi\left(t, x_{2}, \bar{x}_{1}\right)} \chi\left(t, x_{2}, \bar{y}\right)
$$

where $c$ is some absolute constant, $\psi\left(x, x_{1}\right)=-i x \overline{x_{1}} / 2$. We first investigate the critical points of $H$ with respect to $\left(x_{1}, x_{2}\right)$; we estimate ,using [6] page $505(5.30)$

$$
\begin{aligned}
& \operatorname{Im} H\left(t, x, y ; x_{1}, x_{2}\right)+\Phi(x, y) \geq \\
& \quad c\left(\left|x-x_{1}\right|^{2}+\left|\left(x_{2}, \bar{x}_{1}\right)-m_{t}\left(x_{2}, \bar{x}_{1}\right)\right|^{2}+\left|\left(x_{2}, \bar{y}\right)-m_{t}\left(x_{2}, \bar{y}\right)\right|^{2}\right)
\end{aligned}
$$

with the notation $\Phi(x, y)=\varphi_{0}(x)+\varphi_{0}(y)$. We have

$$
\begin{aligned}
& H_{x_{1}}^{\prime}=-\overline{\varphi_{y}^{\prime}\left(t, x_{2}, \bar{x}_{1}\right)}-i / 2 \bar{x}_{1} \\
& H_{\bar{x}_{1}}^{\prime}=-i / 2 x-\overline{\bar{y}_{\bar{y}}^{\prime}\left(t, x_{2}, \bar{x}_{1}\right)}+i / 2 x_{1} \\
& H_{x_{2}}^{\prime}=-\overline{\varphi_{\bar{x}}^{\prime}\left(t, x_{2}, \bar{x}_{1}\right)}+\varphi_{x}^{\prime}\left(t, x_{2}, \bar{y}\right)+i / 2 \bar{x}_{2} \\
& H_{\overline{x_{2}}}^{\prime}=-\overline{\varphi_{x}^{\prime}\left(t, x_{2}, \bar{x}_{1}\right)}+\varphi_{\bar{x}}^{\prime}\left(t, x_{2}, \bar{y}\right)+i / 2 x_{2}
\end{aligned}
$$

It results from these relations that we have a "real" critical point when $x_{1}=x,\left(x_{2}, x_{1}\right) \in \Gamma_{t},\left(x_{2}, \bar{y}\right) \in \Gamma_{t}$, i.e. when $x=y$ the critical point being $x_{1}=x, x_{2}=\Phi_{t}(\bar{x})$. Let $\varepsilon_{1}>0$ a small number to be chosen later.

In the integral 15 , using 18 we can restrict the integration over the set of $\left(x_{1}, x_{2}\right)$ such that $\left|\left(x_{2}, \bar{x}\right)-m_{t}\left(x_{2}, \bar{x}\right)\right|^{2}+\left|\left(x_{2}, \bar{y}\right)-m_{t}\left(x_{2}, \bar{y}\right)\right|^{2} \leq \varepsilon_{0} \lambda^{-1+\varepsilon_{1}}$, $\left|x-x_{1}\right|^{2} \leq \varepsilon_{0} \lambda^{-1+\varepsilon_{1}}$ for some $\varepsilon_{0}$ to be chosen later; we neglect then a term $\mathcal{O}\left(\lambda^{-\infty}\right)$. We deduce from the relations 19 that a zone 
$\left|\varphi_{x}^{\prime}\left(t, x_{2}, \bar{x}\right)-\varphi_{x}^{\prime}\left(t, x_{2}, \bar{y}\right)\right| \geq C \varepsilon_{0}^{1 / 2} \lambda^{\left(-1+\varepsilon_{1}\right) / 2}$ give also a term $\mathcal{O}\left(\lambda^{-\infty}\right)$. As $m_{t}\left(x_{2}, \bar{x}\right)$ and $m_{t}\left(x_{2}, \bar{y}\right) \in \Gamma_{t}$, we have $\Phi_{t}\left(m_{t}\left(x_{2}, \bar{x}\right)_{y}\right)=m_{t}\left(x_{2}, \bar{x}\right)_{x}$ and $\Phi_{t}\left(m_{t}\left(x_{2}, \bar{y}\right)_{y}\right)=m_{t}\left(x_{2}, \bar{y}\right)_{x}, \Phi_{t}$ is a diffeomorphism whose first two derivatives are bounded by some $e^{c t}$, we have then $|x-y| \leq C \varepsilon_{0} e^{c t} \lambda^{\left(-1+\varepsilon_{1}\right) / 2}$.

Let us estimate the Hessian of $H$ at real critical points.

We first make a complexification and we write $\tilde{x}_{1}, \tilde{x}_{2}$ instead of $\bar{x}_{1}, \bar{x}_{2}$, we shall refer to the set $\left\{\tilde{x}_{1}=\bar{x}_{1}, \tilde{x}_{2}=\bar{x}_{2}\right\}$ as the real. Let $\varphi_{1}\left(t, \tilde{x}_{2}, x_{1}\right)$ be an almost analytic extension of $\overline{\varphi\left(t, \overline{\tilde{x}_{2}}, \bar{x}_{1}\right)}$. Let $\tilde{H}$ be an almost analytic function on

$$
\tilde{\Gamma}_{t}=\left\{\left(x, y, x_{1}, \tilde{x}_{1}, x_{2}, \tilde{x}_{2}\right) ;\left(\overline{\tilde{x}_{2}}, \bar{x}_{1}\right) \in \Gamma_{t},\left(x_{2}, \bar{y}\right) \in \Gamma_{t}\right\}
$$

extending $H$. We compute $\nabla^{2} \tilde{H}\left(t, x, x ; x, \bar{x}, \Phi_{t}(\bar{x}), \overline{\Phi_{t}(\bar{x})}\right)$ as the map

$$
\begin{aligned}
& \left(\delta x_{1}, \delta \tilde{x}_{1}, \delta x_{2}, \delta \tilde{x}_{2}\right) \rightarrow \\
& \quad\left(i / 2 \delta \tilde{x}_{1}-\varphi_{1 y y}^{n} \delta x_{1}-\varphi_{1 x y}^{n} \delta \tilde{x}_{2}, i / 2 \delta x_{1}, \varphi_{x x}^{\prime \prime} \delta x_{2}+i / 2 \delta \tilde{x}_{2}\right. \\
& \left.\quad-\varphi_{1 x x}^{n} \delta \tilde{x}_{2}-\varphi_{1 y x}^{\prime} \delta x_{1}+i / 2 \delta x_{2}\right)
\end{aligned}
$$

the computation being simplified by the fact that at such particular points $\nabla^{2} \varphi$ is $C$-linear. This map is invertible and its inverse will have the same norms as the inverse of

$$
\left(\delta x_{2}, \delta \tilde{x}_{2}\right) \rightarrow\left(\varphi_{x x}^{n}\left(t, x_{2}^{c}, \bar{x}\right) \delta x_{2}+i / 2 \delta \tilde{x}_{2},-\bar{\varphi}_{x x}^{\prime \prime}\left(t, x_{2}^{c}, \bar{x}\right) \delta \tilde{x}_{2}+i / 2 \delta x_{2}\right)
$$

with $x_{2}^{c}=\Phi_{t}(\bar{x})$. We have then to estimate the inverse of the map $\bar{\varphi}_{x x}^{\prime \prime}\left(t, x_{2}^{c}, \bar{x}\right) \varphi_{x x}^{\prime \prime}\left(t, x_{2}^{c}, \bar{x}\right)-\frac{1}{4}$. We refer to $([6]$ Proposition $5.2(5.28))$ to obtain

$$
\nabla^{2} \varphi(t, z)=\left(\begin{array}{cc}
\frac{1}{4} b a^{-1} & \frac{i}{2}^{t} a^{-1} \\
-\frac{i}{2} a^{-1} & \frac{1}{4} b^{t} a^{-1}
\end{array}\right)
$$

where $(t, z) \in \Gamma, a$ and $b$ are defined by the relation 23

$$
\left(\delta x-i \delta \xi, \frac{\delta x+i \delta \xi}{2 i}\right)=\left(\begin{array}{cc}
a & b \\
\frac{1}{4} \bar{b} & \bar{a}
\end{array}\right)\left(\delta y-i \delta \eta, \frac{\delta y+i \delta \eta}{2 i}\right)
$$

if $(\delta x, \delta \xi, \delta y, \delta \eta)$ is a tangent vector of $\Lambda_{t}$. We refer to [6] section 5 , page 490. We have

$$
(t, z) \in \Gamma,\left\|\varphi_{x y}^{\prime-1}(t, z)\right\| \leq 2\|a(t, z)\|
$$


Moreover $\emptyset_{x z}^{n} \varphi_{x x}^{n}-\frac{1}{4}=-\frac{1}{4} a^{-1 *} a^{-1}$ as a consequence of the relation $a^{*} a-$ $\frac{1}{4}^{t} b \bar{b}=I$. So the norm of the inverse of the map 21 is $|\operatorname{det} a(t, z)|^{2}$. As computed in $[6]$ the module of $e(t, z)$ at a point $(t, z) \in \Gamma$ is precisely $|\operatorname{det} a(t, z)|^{-1 / 2}$.

This means that in the stationary phase expansion of integral 15 the powers of $\lambda$ and the exponentials decays in time vanish, it remains only the normal $\lambda^{n}$. See [6] section 6.2, page 509-510, relations (6.4) and (6.5).

The condition $\exp (M t) \leq \lambda$ will allow us to give sense to the application of stationary phase expansion with a complex phase function (see [4]) uniformly with respect to $(t, x, y)$.

We need to be more specific in the application of the stationary phase method. We check here some steps with uniform controls in $t$.

In a neighborhond $V=\left\{(x, y) ;|x-y| \leq \varepsilon_{0} e^{-M_{0} t}\right\}$ of the diagonal we have a $C^{\infty} \operatorname{map}(t, x, y) \rightarrow Z_{c}(t, x, y) \in C^{4 n}$, where $Z=\left(x_{1}, \tilde{x}_{1}, x_{2}, \tilde{x}_{2}\right)$, such that $\partial_{z} \tilde{H}\left(t, x, y ; Z_{c}(t, x, y)\right)=0$. The derivatives of this map satisfy some. estimates like $\left|D_{t, x, y}^{\alpha} Z_{c}(t, x, y)\right| \leq C_{\alpha} \exp (c t|\alpha|)$ for some constants $C_{\alpha}$ and $c>0$. Hence we have

$$
\left|Z_{c}(t, x, y)-Z_{c}(t, x, x)\right| \leq C \exp (c t)|x-y|
$$

We can define a symmetric complex matrix $Q(t, x, y)$ such that

$$
\partial_{Z Z}^{2} \tilde{H}\left(t, x, y ; Z_{c}(t, x, y)\right)=i Q^{2}(t, x, y)
$$

which is well defined and smooth since $V$ is connected and simply connected.

We have $\left\|Q^{-1}(t, x, y)\right\| \leq C \exp (c t)$ for some constants $c$ and $C>0$. We have

$$
\begin{aligned}
\operatorname{Im} \tilde{H}(t, x, y ; Z)= & \operatorname{Im}\left(\tilde{H}\left(t, x, y ; Z_{c}(t, x, y)\right)+\right. \\
& 1 / 2 \operatorname{Im} \partial^{2} \tilde{H}_{Z Z}\left(t, x, y ; Z_{c}(t, x, y)\right)\left(Z-Z_{c}(t, x, y)\right)^{2}+ \\
& \mathcal{O}\left(\left(\operatorname{dist}\left(Z_{c}(t, x, y), \text { real }\right)\right)^{\infty}+\left|Z-Z_{c}(t, x, y)\right|^{3}\right)
\end{aligned}
$$

we choose a point $Z$ such that $Z \in$ real $=\left\{x_{1}=\tilde{x}_{1} ; x_{2}=\tilde{x}_{2}\right\}$, then $\operatorname{Im}\left(\widetilde{H}(t, x, y ; Z)+\Phi(x, y) \geq 0\right.$, and $\operatorname{dist}\left(Z_{c}(t, x, y)\right)$, real $) \leq\left|Z-Z_{c}(t, x, y)\right|$. We use then the estimate (2.6) of $[4]$ and we derive

$$
\operatorname{Im} \tilde{H}\left(t, x, y ; Z_{c}(t, x, y)\right)+\Phi(x, y) \geq C e^{-a t} \operatorname{dist}\left(Z_{c}(t, x, y), \text { real }\right)^{2}
$$

This relation shows that the stationary phase expansions are independent of the choice of particular almost analytic extensions but is inadequate to bound the $L^{2}$ norm. 
We shall compute $H_{c}(t, x, y)=\left(t, x, y ; Z_{c}(t, x, y)\right)$ with a Taylor expansion on the diagonal

$$
\begin{aligned}
H_{c}(t, x, y)= & H_{c}(t, x, x)+\nabla_{y} \tilde{H}(t, x, x)(y-x)+1 / 2 \nabla_{y y}^{2} H(t, x, x)(y-x)^{2} \\
& \left.-1 / 2\left(\left(\tilde{H}_{Z Z}^{n}\right)^{-1} \tilde{H}_{Z_{y}}^{n}(y-x), \tilde{H}_{Z y}^{n}(y-x)\right)\right)+\mathcal{O}\left(e^{a t}(x-y)^{3}\right)
\end{aligned}
$$

The second term in 28 is $\varphi_{y}^{\prime}\left(t, x_{2}^{c}, \bar{x}\right)(\bar{y}-\bar{x})=-i / 2 x(\bar{x}-\bar{y})$. The third term is given by $1 / 2 \varphi_{y y}^{\prime \prime}(\bar{y}-\bar{x})^{2}$. $\widetilde{H}_{z y}^{\prime \prime}(y-x)=\left(0,0, \varphi_{x y}^{\prime \prime}(\bar{y}-\bar{x}), 0\right)$. The inverse $\left(\widetilde{H}_{Z Z}^{\prime \prime}\right)^{-1} \delta X=\delta Z$ is given by the relations

$$
\begin{gathered}
\bar{\varphi}_{y x}^{\prime \prime} \delta Z_{2}-\bar{\varphi}_{y y}^{\prime \prime} \delta Z_{1}-i / 2 \delta \widetilde{Z}_{1}=\delta X_{1}, i / 2 \delta Z_{1}=\delta \tilde{X}_{1} \\
\left(\delta Z_{2}, \delta \tilde{Z}_{2}\right)=\left(\begin{array}{cc}
-\left(1 / 4-\bar{\varphi}_{x x}^{n} \varphi_{x x}^{n}\right)^{-1} \bar{\varphi}_{x x}^{n} & -i / 2\left(1 / 4-\bar{\varphi}_{x x}^{n} \varphi_{x x}^{n}\right)^{-1} \\
-i / 2\left(1 / 4-\varphi_{x x}^{n} \bar{\varphi}_{x x}^{n}\right)^{-1} & \left(1 / 4-\varphi_{x x}^{n} \bar{\varphi}_{x x}^{*}\right)^{-1} \varphi_{x x}^{*}
\end{array}\right)\left(\delta X_{2}, \delta \tilde{X}_{2}\right)
\end{gathered}
$$

We make $\delta X=\left(0,0, \varphi_{x y}^{\prime \prime}(\bar{y}-\bar{x}), 0\right)$. The fourth term in 28 is given by $1 / 2\left(\varphi_{x y}^{\prime \prime}(\bar{y}-\bar{x}),\left(1 / 4-\varphi_{x x}^{\prime \prime} \bar{\varphi}_{x x}^{\prime \prime}\right)^{-1} \bar{\varphi}_{x x}^{\prime \prime} \varphi_{x y}^{\prime \prime}(\bar{y}-\bar{x})\right)$. Adding these two terms we have to compute $1 / 2\left(\varphi_{y y}^{n}+\varphi_{y x}^{n}\left(1 / 4-\bar{\varphi}_{x x}^{n} \varphi_{x x}^{n}\right)^{-1} \bar{\varphi}_{x x}^{n} \varphi_{x y}^{n}\right)$. We recall that $\left(1 / 4-\bar{\varphi}_{x x}^{\prime \prime} \varphi_{x x}^{n}\right)=1 / 4 a^{-1 *} a^{-1}$, so $\varphi_{y y}^{n}+\varphi_{y x}^{n}\left(1 / 4-\bar{\varphi}_{x x}^{n} \varphi_{x x}^{n}\right)^{-1} \bar{\varphi}_{x x}^{n} \varphi_{x y}^{n}=$ $1 / 4^{t} b^{t} a^{-1}-1 / 4 a^{-1} a a^{*} b \bar{a}^{-1 t} a^{-1}=0$. We have therefore $H_{c}(t, x, y)=H_{c}(t, x, x)-$ $i / 2 x \bar{y}+i / 2 x \bar{x}$.

We have now to compute $H_{c}(t, x, x),(\partial / \partial t) H_{c}(t, x, x)=-\bar{\varphi}_{t}^{\prime}\left(t, x_{2}^{c}, \bar{x}\right)+$ $\varphi_{t}^{\prime}\left(t, x_{2}^{c}, \bar{x}\right)$ where $x_{2}^{c}=\Phi_{t}(\bar{x})$; on $\Gamma_{t} \varphi_{t}^{\prime}(t, x, y)+p_{1}\left(x, \varphi_{x}^{\prime}(t, x, y)\right)=0$ then Im $\varphi_{t}^{\prime}(t, x, y)=0$; so $H_{c}(t, x, x)=H_{c}(0, x, x)=\psi(x, x)+2 i \operatorname{Im} \psi(x, x)+$ $4 i \varphi_{0}(x)=-\frac{i}{2}|x|^{2}$.

We have obtained

$$
H_{c}(t, x, y)=-\frac{i}{2} x \bar{y}+\mathcal{O}\left(e^{a t}|x-y|^{3}\right)
$$

We deduce from 31 that in $V$,

$$
\operatorname{Im} H_{c}(t, x, y)+\Phi(x, y) \geq C^{-1}|x-y|^{2}
$$

From the usual estimate of $L^{2}$ norms, we obtain the result of Proposition 2. Moreover the relation $e^{M t} \lambda^{-1} \leq 1$ shows that we have an convergent asymptotic development in term of uniform decay in $\lambda$. 
Propasition 3 Let $M$ and $t$ satisfy $e^{M t} \lambda^{-1} \leq 1$, then $E_{t}^{*} E_{t}$ is a pseudodifferential operator of order zero belonging to a class of $S\left(1, g_{\varepsilon}\right)$ (see (3) Chapter 18) where $g_{\varepsilon}=\lambda^{2 \varepsilon}\left(|d x|^{2}+|d \xi|^{2}\right)$, where $\varepsilon>0$ depends on $M$ and on the properties of the flow of $H_{p_{1}}$; when $M \rightarrow \infty, \varepsilon \rightarrow 0$.

Let us recall that a symbol $a \in S\left(1, g_{\varepsilon}\right)$ satisfies uniform estimates

$$
\text { For all multi-indices } \alpha, \beta,\left|D_{x}^{\alpha} D_{\xi}^{\beta} \alpha(x, \xi, \lambda)\right| \leq C_{\alpha, \beta} \lambda^{\varepsilon(|\alpha|+|\beta|)} \text {. }
$$

Proof.

We shall derive this property from Proposition 2 and from the characterization of pseudo-differential operators due to Beals [1]. Let us estimate the $L^{2}$ norm of the first commutators $x^{\alpha} D_{x}^{\beta} E_{t}^{*} E_{t}-E_{t}^{*} E_{t} x^{\alpha} D_{x}^{\beta}$ for $|\alpha|+|\beta|=1$. Using the computations of the proof above we express

$$
\begin{aligned}
x^{\alpha} D_{x}^{\beta} E_{t}^{*} E_{\mathrm{t}} u(x)= & \int x^{\alpha}\left(H_{x c}^{\prime}(t, x, y)\right)^{\beta} f(t, x, y) e^{i \lambda H_{c}(t, x, y)} u(y) d \mu(y) \\
& +\mathcal{O}\left(e^{\alpha t} \lambda^{-1}\right)
\end{aligned}
$$

in this formula $u \in H_{\varphi_{0}}\left(\mathrm{C}^{n}\right)$, the notation $\mathcal{O}$ means that the remainder has the same form but the order of the symbol is lowered. We have $E_{t}^{*} E_{t}\left(x^{\alpha} D_{x}^{\beta} u\right)(x)=\int f(t, x, y) e^{i \lambda H_{c}(t, x, y)} y^{\alpha} D_{y}^{\beta} u(y) d \mu(y)$, we integrate by part in this formula so

$$
\begin{aligned}
E_{t}^{*} E_{t}\left(x^{\alpha} D_{x}^{\beta} u\right)(x)= & (-1)^{|\beta|} \int f(t, x, y) e^{i \lambda H_{c}(t, x, y)} y^{\alpha} \\
& \left(H_{y c}^{\prime}(t, x, y)+i \bar{y} / 2\right)^{\beta} u(y) d \mu(y)+\mathcal{O}\left(e^{a t} \lambda^{-1}\right)(34)
\end{aligned}
$$

We compare 33 and 34 , using 31 we get $H_{x c}^{\prime}(t, x, y)=-i \bar{y} / 2+O\left(e^{a t}(x-y)^{2}\right)$ and $H_{y c}^{\prime}(t, x, y)=\mathcal{O}\left(e^{a t}(x-y)^{2}\right), x-y+\mathcal{O}\left(e^{a t}(x-y)^{2}\right)=H_{y c}^{\prime}(t, x, y)+i / 2 y$.

We need an extra notation to make these integration by parts (more) rigorous. Let $G=\left(\lambda^{-1 / 2}+|x-y|\right)^{-1} e^{C t}\left(|d x|^{2}+|d y|^{2}\right), M_{k}=\left(\lambda^{-1 / 2}+\right.$ $|x-y|)^{-k}$, let $h=H_{c}(t, x, y)+2 i \varphi_{0}(y)$. Assume that an amplitude $f(t, x, y) \in$ $S\left(M_{k}, G\right)$, we note $A(f)$ the integral operator with amplitude $f$ and phase function $h$. Using an integration by parts with the operator $L=\left(\left|h_{\bar{y}}^{\prime}\right|^{2}+\right.$ $\left.\lambda^{-1}\right)^{-1}\left(\bar{h}_{\overline{\mathrm{y}}}^{\prime} \partial / \partial y+1\right)$ and the fact that $u(y)$ is holomorphic we can replace $f$ by $\lambda^{-N}{ }^{t} L^{N}(f)$; so the same operator is given with an amplitude in $S\left(M_{k+2 N} \lambda^{-N} e^{C N t}, G\right)$. We have shown before that if $f \in S\left(M_{k}, G\right)$ $\operatorname{ad}_{X}(A(f))=A\left(f_{1}\right)$ with $f_{1} \in S\left(M_{k} e^{a t} M_{-2}, G\right)$, where $X$ is either $x_{j}$ or $D_{x_{k}}$. So $a d_{X_{1}} \ldots a d_{X_{k}}(A(f))=A\left(f_{k}\right)$ with $f_{k} \in S\left(e^{C k t} \lambda^{-k}, G\right)$. 
We deduce then that $E_{t}^{*} E_{t}$ is a pseudo-differential operator in the class $S\left(1, g_{t}\right)$ where $g_{t}$ is the metric $g_{t}=e^{2 C t}\left(|d x|^{2}+|d \xi|^{2}\right)$.

This result is then optimal with the restriction that we may not have the best constant $C$ and that we consider here spatially homogeneous metrics.

\section{Semi global $L^{2}$ estimates.}

We shall work as in [7] with $L^{2}$ estimates for solutions of the Schrödinger equation $\left(D_{t}+P\left(x, D_{x}\right)\right) u(t)=0$. More precisely

$$
\begin{aligned}
& 2 \lambda I m \int_{T}^{T_{0}}\left(\left(D_{t}+P\left(x, \lambda^{-1} D_{z}\right) u(t), \alpha(t) u(t)\right) d t=\right. \\
& \quad(\alpha(T) u(T), u(T))-\left(\alpha\left(T_{0}\right) u\left(T_{0}\right), u\left(T_{0}\right)\right)+\int_{T}^{T_{0}}(M(t) u(t), u(t)) d t
\end{aligned}
$$

with the notation

$$
\left.M(t)=(\partial \alpha(t) / \partial t)-i \lambda\left[P_{1}, \alpha(t)\right]+2 \lambda \operatorname{Re}\left(\alpha(t) P_{2}\right)\right)
$$

$\alpha(t)\left(x, \lambda^{-1} D_{x}\right)$ is a family of self-adjoint operators to be chosen later, $T_{0}$ will depend only on $\lambda$. We have to make $\left.(\partial \alpha(t) / \partial t)-i \lambda\left[P_{1}, \alpha(t)\right]+2 \lambda \operatorname{Re}\left(\alpha(t) P_{2}\right)\right)$ as large as possible. We choose $\alpha(t)=E_{t} \beta(t) E_{t}^{*}$ where $E_{t}$ has been constructed in section 2 . $\beta(t)$ will be chosen later.

If we note $R(t)=(\partial / \partial t) E_{t}-i \lambda P_{1} E_{t}$, we obtain

$$
\begin{aligned}
\left.(\partial \alpha(t) / \partial t)-i \lambda\left[P_{1}, \alpha(t)\right]+2 \lambda \operatorname{Re}\left(\alpha(t) P_{2}\right)\right) & = \\
E_{t}(\partial \beta(t) / \partial t) E_{t}^{*}+2 \lambda \operatorname{Re}\left(E_{t} \beta(t) E_{t}^{*} P_{2}\right) & +2 \operatorname{Re}\left(R(t) \beta(t) E_{t}^{*}\right)
\end{aligned}
$$

we shall deal later with the last term in 37 . We have written in $5 P_{2}=$ $\left(\left(p_{2}(x, \xi)+\lambda^{-1} \operatorname{Im}\left(p_{0}(x, \xi, \lambda)\right)^{w}\right.\right.$. Let $\beta(t)=\exp (-2 \gamma t)$ where $\operatorname{Im}\left(p_{0}(x, \xi, \lambda) \geq\right.$ $\gamma$. If $Q=P_{2}-\gamma \lambda^{-1}$ the Weyl symbol of $Q$ is non negative. We have to estimate from below the operator $\operatorname{Re}\left(E_{t} E_{t}^{*} Q\right)$, we have proved in Proposition 3 that $E_{t} E_{t}^{*} \in S\left(1, g_{\varepsilon}\right)$, it is then a consequence of the Fefferman-Phong inequality that $\operatorname{Re}\left(E_{t} E_{t}^{*} Q\right) \geq-C \lambda^{-2+2 \varepsilon}$. So from 37 we obtain

$$
(M(t) u(t), u(t)) \geq-C \lambda^{-1+2 \varepsilon} e^{-2 \gamma t}|u(t)|^{2}+2\left(R e\left(R(t) \beta(t) E_{t}^{*}\right) u(t), u(t)\right)
$$

We shall deal later with the last term in 38 . In 35 we shall input $u(t)=u$, so the left hand-side of 35 is an $\mathcal{O}\left(\lambda^{-\infty}\right)$ uniformly in time. 
We make an induction. Let $W$ be an open neighborhood of $\rho_{0}$, we say that $u \in H^{\sigma}(W)$ if for any pseudo-differential operator $\varphi\left(x, \lambda^{-1} D_{x}\right)$ with supp $\varphi \subset W$ u satisfies $|\varphi u| \leq C \lambda^{-\sigma}$. Assume $u \in H^{\sigma}(W)$.

Let $W_{1}, W_{2}$ be two open sets such that $W_{1} \subset \subset W_{2} \subset \subset W$. We use the construction of $[6]$ section 6.3 of a cut-off function $\chi(t, x, y)$ defined by taking an almost analytic extension of the restriction to $\Gamma_{t}$ of the function

$$
\tilde{\chi}(t, y)= \begin{cases}\zeta_{1}(y) \exp \left(-\int_{0}^{t} \frac{\psi}{\zeta^{2}}\left(\Phi_{s}(y)\right) d s\right) & \text { if } \int_{0}^{t} \frac{\phi}{\zeta^{2}}\left(\Phi_{s}(y)\right) d s<\infty \\ 0 & \text { if } \int_{0}^{t} \frac{\psi}{\zeta^{3}}\left(\Phi_{s}(y)\right) d s=\infty\end{cases}
$$

with the notations $\zeta \in C_{0}^{\infty}\left(W_{2}\right), \zeta \equiv 1$ in $\bar{W}_{1} ; \psi \in C_{0}^{\infty}\left(W_{2} \backslash \bar{W}_{1}\right), \psi \equiv 1$ in a neighborhood of $\partial\{x ; \zeta(x)>0\} ; \zeta_{1}(y) \in \mathrm{C}_{0}^{\infty}(\{x ; \zeta(x)>0\})$ is one on a neighborhood of $\vec{W}_{1}$. It was proved in [6] that such constructions give a smooth function whose derivatives are bounded by some $\exp \left(h_{1}(t)\right)$ with $h_{1} \in o(t)$ when $t \rightarrow \infty$. If we add a cut-off function in $\left\{y ;\left|p_{1}(y)\right| \leq e^{-h(t)}\right\}$ the corresponding $\tilde{\chi}$ will be supported in

$$
\Lambda_{W_{2}}=\left\{(t, x, y) ; x=\Phi_{t}(y), \text { for } 0 \leq s \leq t \Phi_{s}(y) \in W_{2},\left|p_{1}(y)\right| \leq c_{2} e^{-h(t)}\right\}
$$

with value 1 on $\Lambda_{W_{1}}$.

In view the lower bound

$$
\operatorname{Im} \varphi(t, x, y)+\Phi(x, y) \geq C^{-1}\left|(x, y)-m_{t}(x, y)\right|^{2}
$$

we shall remain as close to $\Gamma_{t}$ as we wish.

We make $T=0$ and $T_{0}=1 / M \ln \lambda$. The condition in Proposition 3 is satisfied for $0 \leq t \leq T_{0} . \alpha(0)=E_{0} E_{0}^{*}$ is elliptic in $W_{1} . \beta\left(T_{0}\right) E_{T_{0}} E_{T_{0}}^{*}$ is a pseudo-differential operator with wave front set contained in $W_{2}$, belonging to the class $S\left(\varepsilon^{-2 \gamma T_{0}}, g_{\varepsilon}\right)$. We have therefore $\left(\alpha\left(T_{0}\right) u, u\right) \leq C \lambda^{-2 \sigma-2 \gamma / M}$ if $u \in H^{\sigma}(W)$. Using 38 and the fact that $\varepsilon$ is close to 0 , we shall conclude that $H^{\sigma+\gamma / M}(W)$ after taking care of the last term 35 .

The operator $R(t)$ comes from that $E_{t}$ is not an exact solution of the equation $\left(D_{t}+P_{1}\right) E_{t}=0$, which is due to the presence of the $\chi$. The function $\chi$ is itself necessary since we want to localize near $\Lambda(W, h)$ to get our theorem. This analysis has been carried out in [6] section 6.4 . That we wish to say is that the assumption $\overline{\Lambda(W, h)}(p) \cap \partial W \cap O F(u)=0$ implies that $\left|R(t)^{*} u\right|=\mathcal{O}\left(\lambda^{-\infty}\right)$. This kind of troncature are precisely what we need to derive a propagation of singularities theorem from an ordinary $L^{2}$ inequality; as we said above this makes all this machineary necessary. 
Remark 1 The condition $(H)_{3}$ allows to prove a theorem of propagation of singularities with a loss of one derivative, in this sense this condition is sharp.

\section{Part II}

\section{A more precise result in a particular case.}

We shall be able to get a sharper result in a symplectic case analogous to the case treated in [5].

\section{Construction of the stable manifolds.}

In this section we shall use some elements of [10] Appendix A, and [6] Section 4 , we prefer to recall all this material in our proof than to use obscure references to these works.

Let $p(x, \xi)$ be an analytic complex function. Let $p_{0} \in N \cap \mathrm{R}^{2 n}$ where

$$
N=\left\{(x, \xi) \in \mathrm{C}^{2 n} ; p(x, \xi)=d p(x, \xi)=0\right\}
$$

Let $H_{p}=p_{\xi}^{\prime} \partial / \partial x-p_{x}^{\prime} \partial / \partial \xi$ be the hamiltonian field, we mean by bicharacteristic of $p$ the integral curves of the real vector field on $\mathrm{C}^{2 n} H_{p}+\bar{H}_{p}$.

Let

$$
\Lambda_{t}=\left\{(\rho(t, \rho), \rho) ; \rho \in \mathbf{C}^{2 n}, p(\rho)=0\right\} \text { and } \Lambda_{t, \mathbf{R}}=\left\{(\rho(t, \rho), \rho) ; \rho \in \mathbf{R}^{2 n}, p(\rho)=0\right\}
$$

in $43 t \rightarrow \rho(t, \rho)$ is a bicharacteristic curve starting at $\rho$. We shall assume

- $\left(H_{1}\right)$ :

$$
\operatorname{Imp}(\rho) \geq 0 \text { if } \rho \in \mathbf{R}^{2 n} \text {. }
$$

The fundamental matrix is $F_{p}(x, \xi)=d H_{p}(x, \xi)=\left(\begin{array}{cc}p_{\xi x} & p^{\prime \prime}{ }_{\xi \xi} \\ -p_{x x}^{\prime \prime} & -p_{x \xi}^{\prime \prime}\end{array}\right)$.

In the Jordan decomposition of $F_{p}(\rho)$, we note $W_{+}(\rho)=\oplus_{\text {Red }}>0, \lambda \in \operatorname{Spec}\left(F_{\mathrm{p}}\right) V_{\lambda}$, $W_{-}(\rho)=\oplus_{R e \lambda<0, \lambda \in \operatorname{Spec}\left(F_{p}\right)} V_{\lambda} . V_{\lambda}$ are the generalized eigenspaces. 
- $\left(\mathrm{H}_{2}\right)$ : We assume that

$$
C^{2 n}=W_{+}(\rho) \oplus W_{-}(\rho) \oplus W_{0}(\rho) \oplus K \operatorname{er} F_{p}
$$

and the dimensions of these three spaces are constant along $N$.

The assuption $\left(\mathrm{H}_{2}\right)$ means there are no non zero eigenvalue in $i \mathbf{R}$, that there is also no generalized eigenspace relative to zero and that $\operatorname{dim} W_{+}(\rho)=$ $r_{+}, \operatorname{dim} W_{-}(\rho)=r_{-}$are constant. We note respectively by $P_{+}(\rho), P_{-}(\rho)$ and $P_{0}(\rho)$ the corresponding projectors, it follows from our assumption that these maps are analytic.

As $\sigma\left(V_{\lambda}, V_{\mu}\right)=0$ if $\lambda+\mu \neq 0, W_{+}(\rho) \oplus W_{0}(\rho) \subseteq W_{+}(\rho)^{\perp_{\odot}}$, then $r_{+} \leq r_{-}$, then

$$
r_{+}=r_{-}=r \text { and } W_{ \pm}(\rho)^{\perp-}=W_{ \pm}(\rho) \oplus W_{0}(\rho)
$$

- $\left(H_{3}\right)$ : We also assume that in a neighborhod of $\rho_{0}$, there is a constant $C_{0}$ such

$$
\left|H_{p}(\rho)\right| \leq C_{0}\left|\left(I-P_{0}\left(\rho_{0}\right)\right) H_{p}(\rho)\right|
$$

it is a consequence of the assumptions of constant ranks that 47 is independent of $\rho_{0}$.

- $\left(H_{4}\right)$ : We shall assume that on $N^{\prime}=\left\{(x, \xi) \in \mathbf{R}^{2 n} ; \operatorname{Rep}(x, \xi)=\right.$ $d R e p(x, \xi)=0\}$, we have $C^{2 n}=W_{+}^{\prime} \oplus W_{-}^{\prime} \oplus W_{+i}^{\prime} \oplus W_{-i}^{\prime} \oplus K e r F_{R e p}$ where $W_{ \pm}^{\prime}$ are the correponding spaces for $F_{\text {Rep }}$ and $W_{ \pm i}^{\prime}=\oplus_{i \lambda \in S \text { pec }}\left(F_{\text {Rep }}\right), \pm \lambda>0 V_{i \lambda}$.

We suppose also that the quadratic form $[v, \bar{v}]=\frac{1}{i} \sigma(v, \bar{v}) \leq 0$ on $W_{+i}^{\prime}$. This means in fact simply that $V_{0}^{\prime}=K e r F_{\text {Rep }}$ and that there are no no difference of harmonic oscillators in a spectral decomposition of $F_{\text {Rep. }}$. In addition we assume that $N^{\prime}$ is a smooth manifold and that $\operatorname{Ker} \bar{R}_{e p}(\rho)=\mathrm{T}_{\rho} N^{\prime}$.

The first step is to construct stable manifolds for the complex symbol $p$. Let $(x, y)$ be coordinates such that $x \in W_{+}\left(\rho_{0}\right) \oplus W_{0}\left(\rho_{0}\right), y \in W_{-}\left(\rho_{0}\right)$, we split again $x=\left(x^{\prime}, z\right)$ where $x^{\prime} \in W_{+}\left(\rho_{0}\right), z \in W_{0}\left(\rho_{0}\right)$. We note $W^{+}(\rho)=$ $W_{+}(\rho) \oplus W_{0}(\rho)$. Let us note again by $P_{+}(\rho)$ an analytic extension of this function away from $N$.

When we split $\mathbf{C}^{2 n}=W^{+}(\rho) \oplus W_{-}(\rho)$, we have a decomposition of $F_{p}(\rho)=\left(\begin{array}{cc}\alpha & \beta \\ \gamma & \delta\end{array}\right)(\rho)$. We shall split further $\alpha=\left(\begin{array}{ll}\alpha_{0} & \alpha_{1} \\ \alpha_{2} & \alpha_{3}\end{array}\right)$ along $W+(\rho) \oplus$ $W_{0}(\rho)$. 
It is a consequence of the assumptions that in a close neighborhood of $N$,

$\operatorname{Spec}\left(\alpha_{0}\right) \subseteq\{z \in \mathrm{C}, \operatorname{Rez} \geq c\}, \operatorname{Spec}(\delta) \subseteq\{z \in \mathrm{C}, \operatorname{Rez} \leq-c\}$, $\|\beta\| \leq \varepsilon,\|\gamma\| \leq \varepsilon\left\|\alpha_{i}\right\| \leq \varepsilon$ for $1 \leq i \leq 3 ; c>0, \varepsilon$ is small.

Let $\rho$ be a point close to $\rho_{0}$ and $t \rightarrow \rho(t, \rho)$ the bicharacteristic issued from $\rho$. The evolution of tangent vectors is given by the linear differential equation $\frac{d}{d t} v_{t}=F_{p}(\rho(t, \rho)) v_{t}, v_{\left.t\right|_{t=0}=v_{0}}$.

We find a linear map $\varphi_{t}(\rho)$ from $W^{+}\left(\rho_{0}\right)$ to $W_{-}\left(\rho_{0}\right)$ such that the evolution of the space $W^{+}\left(\rho_{0}\right)$ along the flow is given by $W_{t}^{+}(\rho)=\left\{\left(\delta x, \varphi_{t}(\rho) \delta x\right), \delta x \in W^{+}\left(\rho_{0}\right)\right\}$. This is achieved as in [6] by solving the equation

$$
\dot{\varphi}_{t}+\varphi_{t} \alpha-\delta \varphi_{t}+\varphi_{t} \beta \varphi_{t}-\gamma=0,\left.\varphi\right|_{t=0}=0
$$

In view of the relations 48 , which imply that $\operatorname{Spec}(\alpha) \subseteq\{z \in \mathrm{C}, \operatorname{Rez} \geq-\varepsilon\}$ we know that the equation 49 can be solved for $t \geq 0$ and we have $\left\|\varphi_{t}\right\| \leq C \varepsilon$ for some small $\varepsilon$.

We define a suitable norm to construct regions stable under the flow. Let $\alpha_{0}=\alpha\left(\rho_{0}\right)$ and

$$
C_{0}=\int_{-\infty}^{0} \exp \left(t \alpha_{0}^{*}\right) \exp \left(t \alpha_{0}\right) d t, C_{0}>0, C_{0} \alpha_{0}^{*}+\alpha_{0} C_{0}=I d_{W_{+}\left(\rho_{0}\right)}
$$

The restriction of $F_{p}\left(\rho_{0}\right)$ to $\left.W_{(} \rho_{0}\right)$ is expressed by $\delta y \rightarrow \delta_{0}(\delta y), S p e c\left(\delta_{0}\right) \subseteq$ $\{z \in \mathrm{C}, \operatorname{Re} z<-c\}$. We define $D_{0}>0, D_{0} \delta_{0}^{*}+\delta_{0} D_{0}=-I d_{W-\left(\rho_{0}\right)}$.

We note $\left\|v_{x^{\prime}}\right\|^{*}=\left(C_{0} v_{x^{\prime}}, v_{x^{\prime}}\right)^{1 / 2},\left\|v_{x}\right\|^{2}=\left\|v_{x^{\prime}}\right\|^{* 2}+\left|v_{z}\right|^{2},\left\|v_{y}\right\|_{*}=$ $\left(D_{0} v_{y}, v_{y}\right)^{1 / 2}$ and $\|v\|^{2}=\left\|v_{x^{\prime}}\right\|^{* 2}+\left\|v_{y}\right\|_{*}^{2}+\left|v_{z}\right|^{2}$.

We expand

$\frac{d}{d t}\left(\rho_{t}-\rho_{0}\right)=F_{p}\left(\rho_{0}\right)\left(\rho_{t}-\rho_{0}\right)+\mathcal{O}\left(\left(\rho_{t}-\rho_{0}\right)^{2}\right)$ so

$$
\frac{d}{d t}\left\|\rho_{t}-\rho_{0}\right\|^{2}=\left|\left(\rho_{t}-\rho_{0}\right)_{x^{\prime}}\right|^{2}-\left|\left(\rho_{t}-\rho_{0}\right)_{y}\right|^{2}+\mathcal{O}\left(\left(\rho_{t}-\rho_{0}\right)^{3}\right)
$$

Proposition 4 Let $0 \leq f(x) \in \mathrm{C}_{0}^{\infty}\left(\mathbf{R}^{+}\right)$be a function $f \leq \eta$. Let

$$
B\left(\rho_{0}, f\right)=\left\{x \in W^{+}\left(\rho_{0}\right) ;\left\|x^{\prime}\right\|^{*}<f\left(|z|^{2}\right)\right\}
$$

$E\left(\rho_{0}, T, f\right)$ be the region

$$
E\left(\rho_{0}, T, f\right)=\left\{\begin{array}{c}
\rho ; \rho-\rho_{0} \in W^{+}\left(\rho_{0}\right) ; \rho_{t}-\rho_{0} \in B\left(\rho_{0}, f\right) \\
\text { and }\left|\rho_{t}-\rho_{0}\right|<\varepsilon \text { for } 0 \leq t \leq T
\end{array}\right\}
$$


There exist a bounded set of analytic functions $x \in B\left(\rho_{0}, f\right) \rightarrow \lambda(t, x) \in$ $W_{-}\left(\rho_{0}\right)$ such that $E\left(\rho_{0}, T, f\right)$ can be identified with the set

$E^{\prime}\left(\rho_{0}, T, f\right)=\left\{\begin{array}{c}\rho_{i} \rho-\rho_{0} \in W^{+}\left(\rho_{0}\right), \text { for } 0 \leq t \leq T\left|\rho_{t}-\rho_{0}\right|<\varepsilon \text { and } \\ \exists x_{t} \in W^{+}\left(\rho_{0}\right), x_{t} \in B\left(\rho_{0}, f\right) \text { such that } \rho_{t}-\rho_{0}=\left(x_{t}, \lambda\left(t, x_{t}\right)\right)\end{array}\right\}$

This is proved as in [6] by induction on $T$. Let us sketch the proof.

Assume that we have constructed the function $\lambda(t, x)$ for $t=\mathcal{T}_{0}$. We shall prove that it can be extended for some amount in time. The curve $s \rightarrow \rho(s)$ defined by $\rho(s)=\exp \left(-T_{0} H_{p}\right)\left(\rho_{0}+\left(x_{T_{0}}+s \delta x, \lambda\left(T_{0}, x_{T_{0}}+s \delta x\right)\right)\right.$, is a curve in $\rho_{0}+W+\left(\rho_{0}\right)$. It follows from the definition of $\varphi_{t}$ that $(\partial / \partial x) \lambda\left(T_{0}, x\right)=$ $\varphi_{T_{0}}(\rho(0))$, therefore $\left\|(\partial / \partial x) \lambda\left(T_{0}, x\right)\right\| \leq C \varepsilon$. We have also $\lambda\left(T_{0}, 0\right)=0$. In view of the analyticity we derive further controls on all the derivatives.

We note $g(x)=\left\|x^{\prime}\right\|^{*}-f\left(|z|^{2}\right)$, and $\tilde{f}(z)=f\left(|z|^{2}\right)$ with $f(0)>0$.

We define $\psi_{t, T_{0}}(x)=P^{+}\left(\rho_{0}\right)\left(\exp \left(\left(t-T_{0}\right) H_{p}\right)\right)\left(\rho_{0}+\left(x, \lambda\left(T_{0}, x\right)\right)-\rho_{0}\right), \tilde{x}=$ $\psi_{t, T_{0}}(x)$. The map $\psi_{t, T_{0}}$ is close to the identity when $t$ is close to $T_{0}$. We have

$$
\left|\psi_{\tau, T_{0}}(x)-x\right| \leq C\left|\tau-T_{0}\right||x|
$$

We want to prove that $\psi_{t, T_{0}}(x) \in B\left(\rho_{0}, f\right)$ implies $x \in B\left(\rho_{0}, f\right)$. We assume first that $\left|x^{\prime}\right| \geq C^{-1}|x|$.

$$
\begin{aligned}
\frac{d}{d \tau} g\left(\dot{\psi}_{\tau, T_{0}}(x)\right)= & \left\|\dot{\psi}_{\tau, T_{0}}(x)_{x^{\prime}}\right\|^{*-1}<\psi_{\tau, T_{0}}(x)_{x^{\prime}},\left(\frac{d}{d \tau} \psi_{\tau, T_{0}}(x)\right)_{x^{\prime}}> \\
& -\nabla \tilde{f}\left(\left(\psi_{\tau, T_{0}}(x)\right)_{z}\right) \cdot\left(\frac{d}{d \tau} \psi_{\tau, T_{0}}(x)\right)_{z}
\end{aligned}
$$

In view of relation 55 we can replace $\psi_{\tau, T_{0}}(x)$ by $x$ in the first term of 56 modulo $\mathcal{O}\left(\left(\tau-T_{0}\right)\right)$.

We compute $\frac{d}{d \tau} \psi_{\tau, T_{0}}(x)=P^{+}\left(\rho_{0}\right) H_{p}\left(\mu\left(\tau, T_{0}, x\right)\right)$ where $\mu\left(\tau, T_{0}, x\right)=$ $\left.\exp \left(\left(\tau-T_{0}\right) H_{p}\right)\right)\left(\rho_{0}+\left(x, \lambda\left(T_{0}, x\right)\right)\right)$.

Using the estimate $\| \mu\left(\tau, T_{0}, x\right)-\left(\rho_{0}+\left(x, \lambda\left(T_{0}, x\right)\right) \| \leq C\left|\tau-T_{0}\right||x|\right.$, we obtain $\left.H_{p}\left(\mu\left(\tau, T_{0}, x\right)\right)=F_{p}\left(\rho_{0}\right)\left(x, \lambda\left(T_{0}, x\right)\right)\right)+O\left(\left(\tau-T_{0}\right)|x|+|x|^{2}\right)$. Therefore $H_{p}\left(\mu\left(\tau, T_{0}, x\right)\right)=\left(\alpha_{0}\left(\rho_{0}\right) x^{\prime}, 0, \delta\left(\rho_{0}\right) \lambda\left(T_{0}, x\right)\right)+\mathcal{O}\left(\left(\tau-T_{0}\right)|x|+|x|^{2}\right)$. Hence

$$
\left\|\psi_{\tau, T_{0}}(x)_{x^{\prime}}\right\|^{*-1}<\psi_{\tau, T_{0}}(x)_{x^{\prime}},\left(\frac{d}{d \tau} \psi_{\tau, T_{0}}(x)\right)_{x^{\prime}}>\geq C^{-1}\left|x^{\prime}\right|
$$

We want now to estimate the second term in $56,\left(\frac{d}{d \tau} \psi_{7, T_{0}}(x)\right)_{2}=\mathcal{O}((\tau-$ $\left.\left.T_{0}\right)|x|+|x|^{2}\right), \nabla \tilde{f}(z)=O(|z||\nabla f|)$. Therefore $\frac{d}{d r} g\left(\psi_{\tau, T_{0}}(x)\right) \geq C^{-1}\left|x^{\prime}\right|$ when $\left|x^{\prime}\right| \geq C^{-1}|x|$. Then $g\left(\psi_{r, T_{0}}(x)\right) \geq g(x)$, so $x \in B\left(\rho_{0}, f\right)$. 
If on the contrary we have $|z| \geq C\left\|x^{\prime}\right\|^{*}$, then the point $x$ is interior to $B\left(\rho_{0}, f\right)$

We prove now :

Proposition 5 There exist an involutive manifold $E\left(\rho_{0}, \infty\right)$ of codimension $r$, stable under $H_{p}$, contained in $p^{-1}(0)$, such that $\lim _{t \rightarrow \infty} \rho(-t, \rho)$ exist and belongs to $N=\left\{\rho ; p(\rho)=H_{p}(\rho)=0\right\}$ for any bicharacteristic curve issued from a point $\rho \in E\left(\rho_{0}, \infty\right)$.

By the Ascoli's theorem, we know that there is a sequence $t_{j} \rightarrow \infty$, such that the functions $\lambda\left(t_{j}, x\right) \rightarrow \lambda(\infty, x)$.

Let $E^{t}\left(\rho_{0}, f\right)=\exp \left(t H_{p}\right)\left(E\left(\rho_{0}, t, f\right)\right)$.

Let $t \rightarrow \rho(t, \rho)$ be a bicharacteristic curve such that $\rho_{t} \notin E^{t}\left(\rho_{0}, f\right)$, let $\gamma_{t} \in E^{t}\left(\rho_{0}, f\right)$ such that $\rho_{t}-\gamma_{t} \in\left(\mathrm{T}_{\gamma_{t}} E^{t}\left(\rho_{0}, f\right)\right)^{\perp}$, the orthogonality being relative to the \|\| norm, the length of $\rho_{t}-\gamma_{t}$ measures the distance $d\left(\rho_{t}, E^{t}\left(\rho_{0}, f\right)\right)$.

We compute $\frac{d}{d t}\left(\rho_{t}-\gamma_{t}\right)=H_{p}\left(\rho_{t}\right)-\frac{d}{d t} \gamma_{t}$. Let us write $\gamma_{t}=\rho_{0}+$ $\left(x_{t}, \lambda\left(t, x_{t}\right)\right)$; and $\gamma_{t}=\exp \left(t H_{p}\right)\left(\delta_{t}\right), \delta_{t} \in E\left(\rho_{0}, t, f\right)$, so $\frac{d}{d t} \gamma_{t}=H_{p}\left(\gamma_{t}\right)+$ $d\left(\exp \left(t H_{p}\right)\left(\delta_{t}\right)\right) \dot{\delta}_{t}, \dot{\delta}_{t} \in W^{+}\left(\rho_{0}\right)$. Then $\left.d\left(\exp \left(t H_{p}\right)\right)\left(\delta_{t}\right)\right) \dot{\delta}_{t}, \dot{\delta}_{t}=\left(\zeta_{t}, \varphi_{t}\left(\delta_{t}\right) \zeta_{t}\right)$ for some $\zeta_{t} \in W_{+}\left(\rho_{0}\right)$. We have proved above that $\varphi_{t}\left(\delta_{t}\right)=(\partial / \partial x) \lambda\left(t, y_{t}\right)$ where $\exp \left(-t H_{p}\right)\left(\left(\rho_{0}+\left(y_{t}, \lambda\left(t, y_{t}\right)\right)\right)=\delta_{t}\right.$, so $y_{t}=x_{t}$ and $\left(\zeta_{t}, \varphi_{t}\left(\delta_{t}\right) \zeta_{t}\right) \in$ $\mathrm{T}_{\gamma_{t}} E^{t}\left(\rho_{0}, f\right)$.

Therefore $\left.\frac{d}{d t}\left\|\rho_{t}-\gamma_{t}\right\|^{2}=<H_{p}\left(\rho_{t}\right)-H_{p}\left(\gamma_{t}\right), \rho_{t}-\gamma_{t}\right\rangle$, where $<,>$ is the scalar product for \|\| .

$H_{p}\left(\rho_{t}\right)-H_{p}\left(\gamma_{t}\right)=F_{p}\left(\rho_{0}\right)\left(\rho_{t}-\gamma_{t}\right)+\mathcal{O}\left(\left|\left(\rho_{t}-\gamma_{t}\right)\right|^{2}+\left|\left(\rho_{t}-\gamma_{t}\right)\right|\left|\gamma_{t}-\rho_{0}\right|\right)$. As $\left(\rho_{t}-\gamma_{t}\right) \in\left(\mathrm{T}_{\gamma_{t}} E^{t}\left(\rho_{0}, f\right)\right)^{\perp}$, we have the relation

$$
\left(\begin{array}{cc}
C_{0} & 0 \\
0 & I
\end{array}\right)\left(\rho_{t}-\gamma_{t}\right)_{x}+\varphi_{t}\left(\delta_{t}\right)^{*} D_{0}\left(\rho_{t}-\gamma_{t}\right)_{y}=0
$$

We deduce that $\left|\left(\rho_{t}-\gamma_{t}\right)_{x}\right| \leq C \varepsilon\left|\left(\rho_{t}-\gamma_{t}\right)_{y}\right|$.

$$
\begin{gathered}
\left.<F_{p}\left(\rho_{0}\right)\left(\rho_{t}-\gamma_{t}\right), \rho_{t}-\gamma_{t}\right\rangle=\left|\left(\rho_{t}-\gamma_{t}\right)_{x}\right|^{2}-\left|\left(\rho_{t}-\gamma_{t}\right)_{y}\right|^{2} \text {, therefore } \\
d\left(\rho_{t}, E^{t}\left(\rho_{0}, f\right)\right) \leq C \exp \left(-C^{-1} t\right)
\end{gathered}
$$

Let $\rho_{t}=(x, \lambda(t, x)) \in E^{t}\left(\rho_{0}, f\right)$, if $s \leq t$ we write $\rho_{t}=\rho_{s}\left(\rho_{t-s}\right)$, then there exist $y \in W^{+}\left(\rho_{0}\right)$ such that $|(y, \lambda(s, y))-(x, \lambda(t, x))| \leq C \exp \left(-C^{-1} s\right)$, so $|\lambda(t, x)-\lambda(s, x)| \leq C \varepsilon|x-y|+\mid \lambda(s, y))-\lambda(t, x) \mid \leq 2 C \exp \left(-C^{-1} s\right)$. We have therefore proved that $\lambda(t, x) \rightarrow \lambda(\infty, x)$ in the space of holomorphic functions. 
We define

$$
E\left(\rho_{0}, \infty\right)=\left\{\rho_{i} \rho=\rho_{0}+\left(x, \lambda\left(\infty, x_{j}\right) \text { for some } x \in B\left(\rho_{0}, f\right)\right\}\right.
$$

A proof similar shows that

$$
d\left(\rho_{t}, E\left(\rho_{0}, \infty\right)\right) \leq C \exp \left(-C^{-1} t\right) .
$$

Starting from a point $\rho \in E\left(\rho_{0}, \infty\right)$, we prove then that $\lim _{t \rightarrow \infty} \rho(-t, \rho)$ exist.

We prove first that $E\left(\rho_{0}, \infty\right)$ is $H_{p}$ invariant. The tangent space $\mathrm{T}_{\rho} E\left(\rho_{0}, \infty\right)=$ $\left\{\left(\delta x,\left(\frac{\partial}{\partial x}\right) \lambda(\infty, x) \delta x\right)\right\}$ is the limit of the spaces $\left\{\left(\delta x,\left(\frac{\partial}{\partial x}\right) \lambda(t, x) \delta x\right)\right\}$ when $t \rightarrow \infty$. For a point $\rho=\rho_{0}+(x, \lambda(\infty, x))$ we note $y_{t}(x) \in B\left(\rho_{0}, f\right)$ the point defined by $\rho_{0}+(x, \lambda(t, x))=\exp \left(t H_{p}\right)\left(\rho_{0}+\left(y_{t}(x), 0\right) .\left(\frac{\partial}{\partial x}\right) \lambda(t, x)=\varphi_{t}\left(\rho_{0}+\right.\right.$ $\left.\left(y_{t}(x), 0\right)\right), H_{p}(\rho)=m\left(t, \rho_{0}+\left(y_{t}(x), 0\right)\right) v_{t}$, where $v_{t}=H_{p}\left(\rho_{0}+\left(y_{t}(x), 0\right)\right)$, so $w_{t}=m\left(t, \rho_{0}+\left(y_{t}(x), 0\right)\right)\left(\left(v_{t}\right)_{x}, 0\right)=\left(\left(w_{t}\right)_{x}, \varphi_{t}\left(\rho_{0}+\left(y_{t}(x), 0\right)\left(w_{t}\right)_{x}\right)\right) \epsilon$ $\mathrm{T}_{\rho} E^{t}\left(\rho_{0}, t\right)$, the evolution of $\left(v_{t}\right)_{y}$ by $m_{t}$ is an $\mathcal{O}\left(\exp \left(-C^{-1} t\right)\right)$, therefore the distance from $H_{p}(\rho)$ to the space $\left\{\left(\delta x,\left(\frac{\partial}{\partial x}\right) \lambda(t, x) \delta x\right)\right\}$ is also an $\mathcal{O}\left(\exp \left(-C^{-1} t\right)\right)$. So $H_{p}(\rho) \in \mathrm{T}_{p} E\left(\rho_{0}, \infty\right)$.

$$
\frac{d}{d t} \rho(-t, \rho)=-H_{p}(\rho(-t, \rho))=-\left(H_{p}(\rho(-t, \rho))_{x},\left(\frac{\partial}{\partial x}\right) \lambda\left(\infty, x_{t}\right) H_{p}(\rho(-t, \rho))_{x}\right)
$$

where $\rho(-t, \rho)=\rho_{0}+\left(x_{t}, \lambda\left(\infty, x_{t}\right)\right)$. We bound the $H_{p}(\rho(-t, \rho))_{z}$ component of $H_{p}$ by $C_{0}\left(\left|H_{p}(\rho(-t, \rho))_{x^{\prime}}\right|+\left|H_{p}(\rho(-t, \rho))_{y}\right|\right) \leq C_{0}^{\prime}\left|H_{p}(\rho(-t, \rho))_{x^{\prime}}\right|$, using the assumption 47 and 61 . In the backward evolution, the $x^{\prime}$ directions are contractive so

$$
\left|H_{p}(\rho(-t, \rho))_{x}\right| \leq C \exp \left(-C^{-1} t\right)\left|H_{p}(\rho)\right|
$$

this means that $\left|H_{p}(\rho(-t, \rho))\right| \leq C \exp \left(-C^{-1} t\right)\left|H_{p}(\rho)\right|$, then

$\tilde{\rho}=\lim _{t \rightarrow \infty} \rho(-t, \rho)$ exist and belongs to $N^{\prime}=\left\{\rho ; H_{p}(\rho)=0\right\}$. On the connected component of $\rho_{0}$ of $N^{\prime}, p=0$, then $\tilde{\rho} \in N$. But $p(\rho)=p(\rho(-t, \rho))=$ $p(\tilde{\rho})=0$, hence $\left.p\right|_{E(\infty, \infty)}=0$.

$E\left(\rho_{\infty}, \infty\right)$ is a smooth manifold of codimension $r$.

We shall prove that $\mathrm{T}_{\rho}\left(E\left(\rho_{0}, \infty\right)\right)^{\perp_{0}} \subseteq \mathrm{T}_{\rho}\left(E\left(\rho_{0}, \infty\right)\right)_{i} W_{-}\left(\rho_{0}\right)^{\perp_{\sigma}}=$ $W_{-}\left(\rho_{0}\right) \oplus W_{0}\left(\rho_{0}\right)$.

$$
W_{0}\left(\rho_{0}\right)^{\perp_{0}}=\operatorname{Im} F_{p}\left(\rho_{0}\right)=W_{+}\left(\rho_{0}\right) \oplus W_{-}\left(\rho_{0}\right), \text { so }\left.\sigma\right|_{W_{0}\left(\rho_{0}\right)} \text { is non degenerate. }
$$


Let $v_{0} \in \mathrm{T}_{\rho}\left(E\left(\rho_{0}, \infty\right)\right)^{\perp-}, v_{t}=m(t, \rho) v_{0}, v_{t} \in\left(\mathrm{T}_{\rho_{t}}\left(E\left(\rho_{0}, \infty\right)\right)^{\perp_{\sigma}}\right.$. Then for all $\delta x \in W^{+}\left(\rho_{0}\right)$

$$
\sigma\left(\delta x^{\prime},\left(v_{t}\right)_{y}\right)+\sigma\left(\delta z,\left(v_{t}\right)_{z}\right)+\sigma\left(\left(\frac{\partial}{\partial x}\right) \lambda(\infty) \delta x,\left(v_{t}\right)_{x^{\prime}}\right)=0
$$

so $\left|\left(v_{t}\right)_{y}\right|+\left|\left(v_{t}\right)_{z}\right| \leq C \varepsilon\left|\left(v_{t}\right)_{x^{\prime}}\right|$, therefore $\left|v_{t}\right|=\mathcal{O}\left(\exp \left(C^{-1} t\right)\right)$ when $t \rightarrow$ $-\infty$. Therefore $v_{0} \in \mathrm{T}_{\rho}\left(E\left(\rho_{0}, \infty\right)\right) . E\left(\rho_{0}, \infty\right)$ is an involutive manifold. $\sharp$.

We shall prove now $L^{2}$ estimates. This is done by working on the real line only since we look at $\mathrm{C}^{\infty}$ singularities, let us note by $\{f, g\}$ the usual Poisson bracket.

We can now state the main result of this section.

Theorem 2 Let $P\left(x, \lambda^{-1} D_{x}, \lambda\right)=\left(p(x, \xi)+\lambda^{-1} p_{1}(x, \xi, \lambda)\right)^{w_{\lambda}}$ be a pseudodifferential operator such that $p(x, \xi)$ satisfies the assumptions $H_{1}, \ldots, H_{4}$. Let $\lambda_{j}$ be the eigenvalues of $F_{\mathrm{p}}$ with Re $\lambda_{j}>0$ at the points of $N_{\mathbf{R}}=\left\{\rho \in \mathbf{R}^{2 n} ; p(\rho)=d p(\rho)=0\right\}$, we assume that

$$
i p_{1}(x, \xi, \lambda)+\sum_{j}\left(\alpha_{j}+1 / 2\right) \lambda_{j} \neq 0, \text { for all } \alpha_{j} \in \mathbf{N} \text {. }
$$

Let $\gamma(\rho)$ and $c(\rho) \geq 0$ be smooth functions such that $\{p, \gamma\}+c p \geq 0$.

If $\omega$ is a small neighborhood of $\rho_{0} \in N_{\mathbf{R}}$, suppose that $\gamma\left(\rho_{0}\right)>0$ and $\{\gamma>0\} \cap \omega \cap O F(P u)=\emptyset$ and $\{\gamma>0\} \cap \partial \omega \cap O F(u)=\emptyset$, then $\rho_{0} \notin O F(u)$.

Remark 2 It is possible to make a less technical statement in the particular case where $W_{ \pm i}^{\prime}=\{0\}$. In this case $N^{\prime}$ is a smooth symplectic manifold of codimension $2 r^{\prime}$. The involutive manifolds $E_{ \pm}^{\prime}$ have a foliation, we note by $F_{-}(\mu)$ the leaf of $E_{-}^{\prime}$ throught $\mu \in N^{\prime}$. Then the geometric statement of Theorem 2 is : if $F_{-}\left(\rho_{0}\right) \cap \omega \cap O F(P u)=\emptyset$ and $F_{-}\left(\rho_{0}\right) \backslash\left\{\rho_{0}\right\} \cap \omega \cap O F(u)=\emptyset$ then $\rho_{0} \notin O F(u)$.

This remark will be justified below when we will construct appropriate functions $\gamma$. Moreover the presence of the function $c(\rho)$ is needed to have a statement invariant by multipication of $P$ by an operator with a positive symbol.

\section{The energy estimate.}

\subsection{The basic $L^{2}$ inequality.}

The basic $L^{2}$ estimate will be described in the case $I m p_{1}>0$. We shall use microlocal weighted estimates. Let $\gamma(x, \xi) \in \mathrm{C}^{\infty}\left(\mathbf{R}^{2 n}\right)$ a bounded real 
valued function, we note $e_{-}=\left(\lambda^{\gamma(z \xi \xi)}\right)^{w \lambda}$, we write $\mu=\ln \lambda / \lambda$. Let $e_{-\gamma}^{\prime}$ be a parametrix of $e_{\gamma}$. If $A=(a(x, \xi)$ is a pseudo-differential operator with Weyl symbol $a(x, \xi) \in S(1, g)$, then $A_{\gamma}=e_{\gamma}^{\prime} A e_{-\gamma}=\left(a(x, \xi)+i \mu\{a, \gamma\}+O\left(\mu^{2}\right)\right)^{w_{\lambda}}$. We write our operator as $P=\left(p(x, \xi)+\lambda^{-1} p_{1}(x, \xi, \lambda)\right)^{w_{\lambda}}$. Then

$$
P_{\gamma}=\left(p(x, \xi)+i \mu\{p, \gamma\}(x, \xi)+\lambda^{-1} p_{1}(x, \xi, \lambda)+\mathcal{O}\left(\mu^{2}\right)\right)^{w_{\lambda}} \text {. }
$$

We use also a multiplier $M=\left(m^{\prime}+i \mu m^{\prime \prime}\right)$, with two real functions $m^{\prime} \in$ $S(1, g)$ and $m^{*} \in S(1, g)$. We get an energy estimate from the computation of $\operatorname{Im}\left(P_{\gamma} u, M^{*} u\right)$. We have

$$
\operatorname{Im}\left(M P_{\gamma}\right)=\left(m^{\prime} I m p+\mu\left(m^{*} \operatorname{Rep}+m^{\prime}\{\operatorname{Rep}, \gamma\}\right)+\lambda^{-1}\left(m^{\prime} I m p_{1}+\left\{\operatorname{Rep}, m^{\prime}\right\}\right)+\mathcal{O}\left(\mu \lambda^{-1}\right)\right)^{w_{\lambda}} \text {. }
$$

We must make the symbol in 67 positive. The first term $m^{\prime} I m p$ is nonegative if $m^{\prime} \geq 0$. We now concentrate on the second term $m^{\prime \prime} \operatorname{Rep}+m^{\prime}\{\operatorname{Rep}, \gamma\}$. Let $m^{\prime \prime} / m^{\prime}(\rho)=c(\rho)$ be a $C^{\infty}$ function. cRep $+\{R e p, \gamma\}$ is null on $N^{\prime}$ so the best possible choice of $\gamma$ is to make it transversally elliptic on $N^{\prime}$.

$\gamma=\gamma_{0}+\gamma_{1}, c=c_{0}+c_{1}, \gamma_{0}$ and $c_{0}$ are the functions which appears in the statement of Theorem $2, \gamma_{1}$ and $c_{1}$ are constructed below. If $\gamma_{1}$ is null at the second order on $N^{\prime}$, the hessian of $c_{1} \operatorname{Rep}+\left\{\operatorname{Rep}, \gamma_{1}\right\}$ at $\rho \in N^{\prime}$ is given by the fundamental matrix $c_{1}(\rho) F_{\text {Rep }}(\rho)+\left[F_{\text {Rep }}, F_{\gamma_{2}}\right](\rho)$.

We shall localize at points of $N^{\prime}$, let $\rho \in N^{\prime}$ we note

$$
G(\rho)=c_{\mathrm{l}}(\rho) F_{\text {Rep }}(\rho)+\left[F_{\text {Rep }}, F_{\gamma_{1}}\right](\rho) .
$$

The assumption $\left(H_{4}\right)$ implies that at each point in $N^{\prime}$ there is a symplectic basis such that the hessian of $F_{\text {Rep }}$ is a sum of terms

(i) $Q(x, \xi)=a x . \xi$, with $\operatorname{Spec}(a) \subseteq\{z \in \mathrm{C} ; \operatorname{Rez}>0\}$

(ii) $Q(x, \xi)=\alpha\left(x^{2}+\xi^{2}\right)$, with $\alpha>0$.

(iii) $Q(x, \xi)=0$.

We shall find appropriate quadratic form $\gamma_{1}$ and constant $c_{1}$ at $\rho$ so that $G$ in 68 is positive and piece them together. If we are in case (i), we chose $\gamma_{1}(x, \xi)=(\alpha x, x)-(\beta \xi, \xi) \alpha$ and $\beta$ are two positive matrices so that $\sigma\left((x, \xi),\left[F_{R e p}, F_{\gamma_{1}}\right](x, \xi)\right) \geq 1 / C\left(x^{2}+\xi^{2}\right) ;$ any $c_{1}>0$ will fit.

If we are in case (ii), we take $\gamma_{1}(x, \xi)=-k\left(x^{2}+\xi^{2}\right)$ with $k>0$ and small with respect to $c_{1}$.

In case (iii) $\gamma_{1}=0$. Therefore we can construct functions $\gamma(\rho)$ and $c(\rho)$, such that

$$
(c \operatorname{Rep}+\{\operatorname{Rep}, \gamma\})(\rho) \geq C^{-1} d\left(\rho, N^{\prime}\right)^{2}
$$


Moreover if $\gamma_{1}$ is small with respect to $\gamma_{0}$ we shall have $\{\gamma>0\} \cap \partial \omega \cap$ $O F(u) \subseteq\left\{\gamma_{0}>-\varepsilon\right\} \cap \partial w \cap O F(u)=\emptyset$.

We choose $m^{\prime}(\rho)=\varphi(\rho)^{2}$, where $\varphi$ is a $C^{\infty}$ function supported by $\omega$. $I m p_{1}>0$ is positive, while $\left\{\right.$ Rep, $\left.m^{\prime}\right\}$ is supported near $\partial \omega$. We derive the estimate

$\operatorname{Im}\left(P_{\gamma} u, M^{*} u\right) \geq c \mu\left(\sum_{j}\left|v_{j}(\varphi u)\right|^{2}\right)+c \lambda^{-1}|\varphi u|^{2}+\mathcal{O}\left(\lambda^{-1}\right)|\psi u|^{2}+\mathcal{O}\left(\lambda^{-1} \mu\right)|u|^{2}$

the $v_{j}$ form a set of equations of $N^{\prime}, \psi$ is supported near $\partial \omega$. We replace $u$ by $e_{\gamma}^{\prime} u$ and we note $M_{\gamma}=e_{\gamma}^{\prime} M e_{\gamma}^{j}$. In the following the third term in 70 could be neglected since $O F(u) \cap \partial \omega \cap\{\gamma>0\}=0$.

We introduce the additionnal notation : let $m$ be an order function and $g$ a metric a symbol $a(x, \xi) \in \tilde{S}(m, g)$ if it is the sum of a symbol in $S(m, g)$ supported by a neighborhod of the support of $\varphi$ and a symbol of order $-\infty$. In the following $m$ will have the form $\lambda^{m}(\ln \lambda)^{p}$ and $g=g_{0}$ or $m=\lambda^{\gamma}(\ln \lambda)^{p}$ and $g^{\prime}=(\ln \lambda)^{2} g_{0}$. Then we have

$$
\left.\operatorname{Im}\left(P u, M_{\gamma}^{*} u\right) \geq c\left(\mu \sum_{j}\left|v_{j} \varphi e_{\gamma}^{\prime} u\right|^{2}\right)+\lambda^{-1}\left|\varphi e_{\gamma}^{\prime} u\right|^{2}\right)+\left(R_{2 \gamma-2,2} u, u\right) .
$$

with $R \in \tilde{S}\left(\lambda^{2 \gamma-2}(\ln \lambda)^{2}, g^{\prime}\right)$. We shall use the notation $|u|_{\gamma}=\left|e_{\gamma}^{\prime} u\right|$.

\subsection{Concatenations.}

We move the subprincipal symbol using multiplication by non elliptic operators, this is named concatenations.

$$
\operatorname{Im}\left(J U_{N} P u, J M_{\gamma}^{*} U_{N} u\right)=\operatorname{Im}\left(\left[J U_{N}, P\right] u, J M_{\gamma}^{*} U_{N} u\right)++\operatorname{Im}\left(P J U_{N}, M_{\gamma}^{*} J U_{N} u\right)
$$

where $U_{N} u=\left(U_{\alpha} u\right)_{|\alpha|=N}$ and $U_{\alpha}=\left(u_{1}^{w_{\lambda}}\right)^{\alpha_{1}} \cdots\left(u_{r}^{w_{\lambda}}\right)^{\alpha_{+}}, J$ is a linear operator in the space $\mathrm{C}^{N^{\prime}}$ of multi-indices of length $N$. We can apply inequality 71 to the second term of $\mathbf{7 2}$.

We compute the commutator $\left[P, U_{N}\right]$. Let $P_{0}$ be the principal part of $P$, i.e. $P_{0}=(p)^{w_{\lambda}}=\sum_{1 \leq j \leq r}\left(p_{j} u_{j}\right)^{w_{\lambda}}$ and $P=P_{0}+i \lambda^{-1}\left(p_{1}\right)^{w_{\lambda}}$, $p_{1}$ is the sub principal symbol.

$$
\left[U_{\alpha}, P_{0}\right]=\sum_{1 \leq j \leq r, p+q=\alpha_{j}-1}\left(u_{1}^{w_{\lambda}}\right)^{\alpha_{1}} \cdots\left(u_{j}^{w_{\lambda}}\right)^{p}\left[u_{j}^{w_{\lambda}}, P_{0}\right]\left(u_{j}^{w_{\lambda}}\right)^{q} \cdots\left(u_{r}^{w_{\lambda}}\right)^{\alpha_{r}} .
$$


$\operatorname{But}\left[u_{j}^{w_{\lambda}}, P_{0}\right]=\sum_{1 \leq k \leq r}\left[u_{j}^{w_{\lambda}},\left(p_{k} u_{k}\right)^{w_{\lambda}}\right],\left(p_{k} u_{k}\right)^{w_{\lambda}}=p_{k}^{w_{\lambda}} u_{k}^{w_{\lambda}}-\frac{1}{2 i \lambda}\left\{p_{k}, u_{k}\right\}^{w_{\lambda}}+$ $\mathcal{O}\left(\lambda^{-2}\right)$, so $\left[u_{j}^{w_{\lambda}}, P_{0}\right]=\sum_{1 \leq k \leq r}\left[u_{j}^{w_{\lambda}}, p_{k}^{w_{\lambda}}\right] u_{k}^{w_{\lambda}}+\mathcal{O}\left(\lambda^{-2}\right)$.

We deduce then

$$
\begin{aligned}
{\left[U_{\alpha}, P_{0}\right]=} & \\
& \sum_{1 \leq k \leq r, 1 \leq j \leq r} \frac{1}{i \lambda}\left\{u_{j}, p_{k}\right\}^{w_{\lambda}} \alpha_{j}\left(u_{1}^{w_{\lambda}}\right)^{\alpha_{1}} \cdots\left(u_{k}^{w_{\lambda}}\right)^{\alpha_{k+1}} \cdots\left(u_{j}^{w_{\lambda}}\right)^{\alpha_{j}-1} \cdots\left(u_{r}^{w_{\lambda}}\right)^{\alpha_{r}} \\
& +\sum_{\beta<\alpha}\left(c_{\alpha, \beta}\right)^{w_{\lambda}} U_{\beta}
\end{aligned}
$$

where $c_{\alpha, \beta}$ are symbols of degree $-1-|\alpha|+|\beta|$.

We know that $\operatorname{Spec}\left(\left\{p_{k}, u_{j}\right\}\right) \subset\{z \in \mathrm{C}, \operatorname{Re} z>c\}$.

The operator $\left(z_{\alpha}\right) \rightarrow\left(\alpha_{j} z_{\alpha-(j)+(k)}\right)$ is algebraically the operator $z_{k} \frac{\partial}{\partial z_{j}}$. With the notation $a_{j, k}=\left\{u_{j}, p_{k}\right\}$, and

$$
\left(\mathcal{A}_{N} U\right)_{\alpha}=\sum_{\alpha=\beta-(j)+(k), 1 \leq j, k \leq r} a_{k, j} \beta_{j} U_{\beta}
$$

we have

$$
\left[U_{N}, P_{0}\right]=i \lambda^{-1}\left(\mathcal{A}_{N} U_{N}\right)+\left(\sum_{\beta<\alpha}\left(c_{\alpha, \beta}\right)^{w_{\lambda}} U_{\beta}\right)_{\alpha}
$$

The same result will hold for $P$ since $P_{1}$ will contribute to the second term in 75 .

We construct the linear operator $J$, such that $J \mathcal{A}_{N}\left(\rho_{0}\right) J^{-1}=\left(\left(\sum_{j} \alpha_{j} \lambda_{j}\right) \delta_{\alpha, \beta}\right)+$ $o(N)$, the contribution $o(N)$ is due to that eventually $a\left(\rho_{0}\right)$ cannot be made diagonal. We see that the self-adjoint part of operator $M_{\gamma} J A_{N} J^{-1}$ is positive elliptic. Using the Gärding inequality for systems we have

$$
\operatorname{Im}\left(J A_{N} U_{N} u, J M_{\gamma}^{*} U_{N} u\right) \geq c N\left|\varphi^{w_{\lambda}} e_{\gamma}^{\prime} J U_{N} u\right|^{2}-C_{N}\left(R_{2 \gamma-1,2} U_{N} u, U_{N} u\right)
$$

where $R_{2 \gamma-1,2} \in \bar{S}\left(\lambda^{2 \gamma-1}(\ln \lambda)^{2}, g^{\prime}\right)$.

We estimate

$$
\begin{aligned}
& \left.\operatorname{Im}\left(J \mid U_{N}, P\right] u, J M_{\gamma}^{*} U_{N} u\right) \geq \\
& \quad c N \lambda^{-1}\left|\varphi e_{\gamma}^{\prime} J U_{N} u\right|^{2}+\left(R_{2 \gamma-2,2}^{(N)} U_{N} u, U_{N} u\right)+\sum_{i<N} \operatorname{Re}\left(J C_{N, l} U_{l} u, M_{\gamma}^{*} J_{N} u\right)
\end{aligned}
$$


where $R^{(N)} \in \tilde{S}\left(\lambda^{2 \gamma-2}(\ln \lambda)^{2}, g^{\prime}\right)$, the $C_{N, l}$ are operators of order $-1-N+l$. We estimate the third term in 77 . The operator $M_{\gamma}=e_{\gamma}^{\prime}\left(\varphi^{w_{\lambda}}\right)^{2} e_{\gamma}^{\prime}+R_{2 \gamma-1,2}$, then

$$
\begin{aligned}
& \operatorname{Re}\left(M_{\gamma} J C_{N, l} U_{l} u, J U_{N} u\right) \leq \\
& \quad \varepsilon \lambda^{-1}\left|\varphi^{w_{\lambda}} e_{\gamma}^{\prime} J U_{N} u\right|^{2}+C_{N, \varepsilon} \lambda^{-1-2 N+2 l}\left|U_{l} u\right|_{\gamma}^{2}+C_{N} \lambda^{-3}(\ln \lambda)^{4}\left|U_{N} u\right|_{\gamma}^{2}
\end{aligned}
$$

So we get

$$
\begin{aligned}
& \operatorname{Im}\left(J\left[U_{N}, P\right] u, J M_{\gamma}^{*} u\right) \geq \\
& \left.\quad c N \lambda^{-1}\left|\varphi e_{\gamma}^{\prime} J U_{N} u\right|^{2}+\left(R_{2 \gamma-2,2}^{(N)} U_{N} u, U_{N} u\right)+\sum_{l<N} R_{2 \gamma-2 N+2 u}^{(N, l)} U_{l} u, U_{l} u\right)
\end{aligned}
$$

If we chose $N$ such that $I m p_{1}+c N>0$, using 71,72 and 79 we obtain

$$
\begin{aligned}
& \operatorname{Im}\left(J U_{N} P u, J M_{\gamma}^{*} U_{N} u\right) \geq \\
& \quad c N \lambda^{-1}\left|\varphi^{w_{\lambda}} e_{\gamma}^{\prime} J U_{N} u\right|^{2}-C \lambda^{-2}(\ln \lambda)^{4}\left|\varphi_{1}^{w_{\lambda}} e_{\gamma}^{\prime} U_{N} u\right|^{2} \\
& \quad-C_{N} \sum_{0 \leq k \leq N-1} \lambda^{-1-2 N+2 k}\left|\varphi_{1}^{w_{\lambda}} e_{\gamma}^{\prime} U_{k} u\right|^{2}+\mathcal{O}\left(\lambda^{-\infty}\right)
\end{aligned}
$$

where $\varphi_{1}$ is a function supported by a neighborhod of suppp.

As in [5] the proof is based on a recurrence on the $H^{s}$ regularity of the $U_{k} u$ in the domain $\{\gamma(x, \xi)>0\} \cap \omega$.

We must modify the Proposition 1 of [5] to take care of the last terms in 80 . We shall estimate $c_{k}=\sum_{0 \leq l \leq k} \lambda^{l}\left|e_{\gamma}^{\prime} \varphi^{w_{\lambda}} U_{k} u\right|$ by $c_{N}, N$ is now fixed. To do that we use the equation

$$
P u=f=\sum_{1 \leq j \leq \leq r} p_{j}^{w_{\lambda}} u_{j}^{w_{\lambda}}(u)+\left(\frac{1}{2 i \lambda}\left\{u_{j}, p_{j}\right\}+\lambda^{-1} p_{1}+O\left(\lambda^{-2}\right)\right)^{w_{\lambda}}(u)
$$

We note

$$
p_{1}^{\prime}=\frac{1}{2 i} \sum_{1 \leq j \leq r}\left\{u_{j}, p_{j}\right\}+p_{1}
$$

We use as above commutators with the operators $U_{k}$; from formula 75 we obtain

$$
\begin{aligned}
U_{n} f=U_{n} P u= & i \lambda^{-1} \mathcal{A}_{n} U_{n}(u)+\sum_{0 \leq j \leq n-1} Q_{n j} U_{j}(u) \\
& +\sum_{1 \leq j \leq \leq r} p_{j}^{w \lambda} u_{j} U_{n}(u)+\lambda^{-1} p_{1}^{\prime w} U_{n}(u)+\mathcal{O}\left(\lambda^{-2}\right)(u)
\end{aligned}
$$


The $Q_{n, j}$ are operators of order $-1-n+j$.

It is a consequence of the assumptions that the matrix $\mathcal{A}_{n}^{\prime}=\mathcal{A}_{n}+I d_{\mathrm{C}^{\prime}} p_{1}^{\prime}$ is invertible for any $n$ in a neighborhod of $\rho_{0}$. Let $\mathcal{B}_{n}$ an operator of order $\gamma$ such that $B_{n} \mathcal{A}_{n}^{\prime}=\varphi_{0} e_{\gamma}^{\prime} I d+\mathcal{O}\left(\lambda^{-\infty}\right)$. We apply $\mathcal{B}_{n}$ on the members of equation 83

$$
\mathcal{B}_{n}(f)=i \lambda \varphi_{0}^{\omega_{\lambda}} e_{\gamma}^{\prime} U_{n}+\sum_{1 \leq j \leq r} B_{n} P_{j}\left(U_{n u} u\right)+\sum_{l<n} \mathcal{B}_{n} Q_{n, j} U_{j} u
$$

We multiply both members of 84 by $\varphi^{w_{\lambda}}$, using $\varphi^{w_{\lambda}} \varphi_{0}^{w_{\lambda}}=\varphi^{w_{\lambda}}+\mathcal{O}\left(\lambda^{-\infty}\right)$, $\left[\mathcal{B}_{n} P_{j}, \varphi^{w_{\lambda}}\right] \in \tilde{S}\left(\lambda^{\gamma-1}(\ln \lambda), g^{\prime}\right)$ and $\left[\mathcal{B}_{n} Q_{n, j}, \varphi^{w_{\lambda}}\right] \in \tilde{S}\left(\lambda^{\gamma-2-n+j}(\ln \lambda), g^{\prime}\right)$ we obtain

$$
\begin{aligned}
& \left|\varphi^{w_{\lambda}} e_{\gamma}^{\prime} U_{n} u\right| \leq \\
& C\left(o(1) \sum_{1 \leq j \leq r}\left|\varphi^{w_{\lambda}} u_{j} U_{n} u\right|_{\gamma+1}+\sum_{l<n}\left|\varphi^{w_{\lambda}} U_{l} u\right|_{\gamma-n+l}+\sum_{l \leq n+1}\left|\varphi_{1}^{w_{\lambda}} U_{l} u\right|_{\gamma-n+l, 1}\right) \\
& \quad+\mathcal{O}\left(\lambda^{-\infty}\right)
\end{aligned}
$$

This justify the notations $c_{n}=\sum_{j \leq n} \lambda^{j}\left|e_{\gamma}^{\prime} \varphi^{w_{\lambda}} u\right|$ and $d_{n}=(\ln \lambda) \sum_{j \leq n} \lambda^{j}\left|e_{\gamma}^{\prime} \varphi_{1}^{w_{\lambda}} u\right|$. We have proved

$$
c_{n} \leq o(1) c_{n+1}+k_{0} \sum_{j<n} c_{j}+k_{1} d_{n+1}+\mathcal{O}\left(\lambda^{-\infty}\right)
$$

$k_{0}, k_{1}$ are some constant.

The basic idea of Propsition 1 of [5] is to derive from 86 an upper bound of the $c_{j}$ for $0 \leq j \leq N-1$ by $c_{N}$, where $N$ is an integer choosen large enough with respect to the imaginary part of $p_{1}$. We shall need eventually to shrink $\omega$ accordingly. The $d_{j}$ are controlled by using the steps of this recurrence. So we obtain by recurrence the smoothness of $u$ in the domain $\{(x, \xi) ; \gamma(x, \xi)>0\} \cap \omega$.

Now we can finish the proof as in [5].

\section{References}

[1] R. Beals, Characterization of pseudo differential operators, Duke Mathematical Journal 42, (1975), 1-42

[2] J.M. Bony and N. Lerner, Quantification asymptotique et microlocalisations d'ordre supérieur I, Ann. ENS, 4 série, 22, (1989) 377-433. 
[3] L. Hörmander, The Analysis of Linear Partial Differential Operators III, Springer-Verlag, 274, 1985.

[4] A. Melin and J. Sjöstrand, Fourier Integral Operators with complexvalued phase function, Lecture Notes in Mathematics, Springer-Verlag, $459,1975$.

[5] B. Lascar and R. Lascar, Propagation des singularités pour des opérateurs dont la matrice fondamentale contient des valeurs propres non purement imaginaires. Comm. in Partial Differential Equations, 17 (3\&4), (1992), 437-446.

[6] B. Lascar and J. Sjöstrand, Equation de Schrödinger et propagation pour des o.p.d. à caractéristiques réelles de multiplicité variable II, Comm. in Partial Differential Equations, 10 (5), (1985), 467-523.

[7] R. Lascar, Propagation des singularités pour des opérateurs à symboles complexes, To appear, Comptes Rendus Acad. Sciences, 1992.

[8] N. Lerner, A non İocally solvable pseudo-diffenential operator which satisfies the condition $(\Psi)$. To appear in Annals of Maths.

[9] J. Sjöstrand, Singularités analytiques microlocale, Astérisque 95, 1984.

[10] J. Sjöstrand, Analytic wave front sets and operators with multiple characteristics, Hokkaido Mathematical Journal 12 (1983) 392-433. 